\title{
The small-time Chung-Wichura law for Lévy processes with non-vanishing Brownian component
}

\author{
Boris Buchmann • Ross Maller
}

Received: 5 April 2009 / Revised: 27 October 2009 / Published online: 11 December 2009

(C) Springer-Verlag 2009

\begin{abstract}
We give a "small time" functional version of Chung's "other" law of the iterated logarithm for Lévy processes with non-vanishing Brownian component. This is an analogue of the "other" law of the iterated logarithm at "large times" for Lévy processes and random walks with finite variance, as extended to a functional version by Wichura. As one of many possible applications, we mention a functional law for a two-sided passage time process.
\end{abstract}

Keywords Lévy process - Local behaviour - Almost sure convergence $\cdot$ Iterated logarithm laws · Other law of the iterated logarithm · Cluster sets · Functional limit theorem

Mathematics Subject Classification (2000) $\quad 60 \mathrm{G} 51 \cdot 60 \mathrm{~F} 15 \cdot 60 \mathrm{~F} 17 \cdot 60 \mathrm{~F} 05$. $60 \mathrm{~J} 65 \cdot 60 \mathrm{~J} 75$

\section{Introduction}

The "other law of the iterated logarithm", as a large-sample theorem for a random walk $S_{n}$, has a long and distinguished history. It was originally conceived as a counterpart

This research was partially supported by ARC grants DP0664603 and DP0988483.

B. Buchmann $(\varangle)$

School of Mathematical Sciences, Monash University, Clayton Campus, Clayton, VIC 3800, Australia e-mail: Boris.Buchmann@sci.monash.edu

R. Maller

Centre for Mathematics and its Applications, School of Finance and Applied Statistics,

Australian National University, Canberra, ACT, Australia

e-mail: Ross.Maller@anu.edu.au 
to the "usual" law of the iterated logarithm by Chung [8], and proved in the form

$$
\liminf _{n \uparrow \infty} \frac{\max _{1 \leq j \leq n}\left|S_{j}\right|}{\sqrt{n / \log \log n}}=\frac{\pi \sqrt{E S_{1}^{2}}}{\sqrt{8}}, \quad \text { a.s. }
$$

under the assumption that the i.i.d. increments of $S_{n}$ have a finite absolute third moment and expectation 0 . This was subsequently improved to a second moment assumption by Jain and Pruitt [14].

There have been many extensions and spin-off results related to this "other law". Among them we mention a converse by Csáki [9], a definitive upper/lower class integral test by Einmahl [11], and very general formulations by Einmahl and Mason [12] and Kesten [16]. In [8], Chung also showed a corresponding large time result for Brownian motion. Csáki [10] found an interesting connection between Chung's law for Brownian motion and the approximation error in Strassen's law of the iterated logarithm; see also Gorn and Lifshits [13]. For an excellent historical account of laws of iterated logarithms we refer to Bingham [5].

In an apparently unpublished investigation, Wichura [36] is cited, e.g., in [18,26], as obtaining functional versions of (1.1), also replacing $S$ by a Brownian motion. As further and more recent spin-offs of Wichura's result we mention Mueller ([26,27]), N'Zi, Rémillard and Theodorescu [28] and Kuelbs and Li [18], and references therein.

All of the latter papers deal with Gaussian processes or functionals of them. Extensions of Wichura's functional law to Lévy processes have been investigated by Chen, Kuelbs and Li [7] (for symmetric stable processes) and Rushton [29] (random walks as well as Lévy processes in the domain of normal attraction of a stable process). These are large time results. Our aim in this paper is to obtain a version of Wichura's functional law for Lévy processes as the time parameter becomes small. They can be regarded as amplifications of small time laws of the iterated logarithm and generalised laws of the iterated logarithm for Lévy processes. See Maller [24] for a discussion of some of these, and of the small time "square root" functional limit theorem of Bertoin, Doney and Maller [4] and Buchmann, Maller and Szimayer [6]. Bertoin [3], p.88, and Sato [30], p.351, give other relevant "small time" almost sure boundedness results for Lévy processes.

Many of our techniques are gleaned from this literature, as well as from the large time literature, especially [7] and [29], but the small time investigations do require the development of some new methods, and also suggest some interesting new areas of research, as outlined in Remark 2.1 below.

\section{Main result}

Our notations are as follows. Let $(\Omega, \mathcal{F}, P)$ be a probability space carrying a Lévy process $(X(t))_{t \geq 0}=(X(t, \omega))_{t \geq 0}, \omega \in \Omega$, with $X(0)=0$ and canonical triplet $\left(\gamma, \sigma^{2}, \Pi\right)$, where $\gamma \in \mathbb{R}, \sigma^{2} \geq 0$, and $\Pi$ is a nonnegative measure on $\mathbb{R}$ satisfying $\Pi(\{0\})=0$ and 


$$
\int_{\mathbb{R}}\left(x^{2} \wedge 1\right) \Pi(\mathrm{d} x)<\infty
$$

The finite dimensional distributions of $X$ are determined by the distribution of $X(1)$ via the celebrated Lévy-Khintchine formula, which states that $E e^{\mathrm{i} \theta X(t)}=e^{t \Psi(\theta)}$, where

$$
\Psi(\theta)=\mathrm{i} \gamma \theta-\frac{\sigma^{2} \theta^{2}}{2}+\int_{\mathbb{R}}\left(e^{\mathrm{i} \theta x}-1-\mathrm{i} \theta x 1_{|x| \leq 1}\right) \Pi(\mathrm{d} x),
$$

for $t>0$ and $\theta \in \mathbb{R}$. Without loss of generality, we may assume throughout that, for all $\omega \in \Omega$, the sample paths $t \mapsto X(t, \omega)$ are càdlàg functions, i.e., right-continuous with left limits. See Bertoin [3] and Sato [30] for basic properties of Lévy processes.

Let

$$
\ell_{2}(t)= \begin{cases}\log |\log t|, & t \leq e^{-e} \\ 1, & t>e^{-e}\end{cases}
$$

Throughout, for $t \geq 0$ and a locally bounded function $h:[0, \infty) \rightarrow \mathbb{R}$, we use the notation $\|h\|_{t}=\sup _{0 \leq s \leq t}|h(s)|$.

For $t>0$, we define

$$
Z_{t}(s)=\frac{\|X\|_{s t}}{\sqrt{t / \ell_{2}(t)}}, \quad s \geq 0
$$

We are interested in the almost sure behaviour of the family of processes $Z=\left(Z_{t}\right)_{t>0}$, defined on the positive reals $[0, \infty)$ and indexed by $t>0$, for small values of $t$.

Our functional set-up is as follows: let $\mathcal{L}$ be the space of functions $f:[0, \infty) \rightarrow$ $[0,1]$ such that $f(0)=0, f$ is nondecreasing and right continuous on $(0, \infty)$ and $\lim _{s \rightarrow \infty} f(s)=1$. We endow $\mathcal{L}$ with the Lévy metric:

$$
d_{\mathcal{L}}(f, g)=\inf \left\{\varepsilon>0: f\left((s-\varepsilon)^{+}\right)-\varepsilon \leq g(s) \leq f(s+\varepsilon)+\varepsilon, \quad s \geq 0\right\} .
$$

Recall that we have $f_{n} \rightarrow f$ in $\left(\mathcal{L}, d_{\mathcal{L}}\right)$ as $n \rightarrow \infty$, if and only if $f_{n}(s) \rightarrow f(s)$, as $n \rightarrow \infty$, for all continuity points $s$ of $f$.

Let $\mathcal{M}$ be the space of functions $f:[0, \infty) \rightarrow[0, \infty]$ such that $f(0)=0$, $f$ is nondecreasing and right continuous on $(0, \infty)$ and $\lim _{s \rightarrow \infty} f(s)=\infty$. Let $\theta(s)=s /(1+s), 0 \leq s<\infty$, with $\theta(\infty)=1$, and define

$$
f^{*}(s)=\theta(f(s)), \quad f \in \mathcal{M}, \quad s \geq 0 .
$$

Then $f^{*} \in \mathcal{L}$, provided that $f \in \mathcal{M}$, and we consider the topology on $\mathcal{M}$ induced by the metric

$$
d_{\mathcal{M}}(f, g)=d_{\mathcal{L}}\left(f^{*}, g^{*}\right),
$$


which makes $\left(\mathcal{M}, d_{\mathcal{M}}\right)$ a complete and separable metric space. Further, we have $f_{n} \rightarrow f$ in $\left(\mathcal{M}, d_{\mathcal{M}}\right)$, as $n \rightarrow \infty$, if and only if $f_{n}(s) \rightarrow f(s)$, as $n \rightarrow \infty$, for all continuity points $s$ of $f$.

For a family of random elements $Y=\left(Y_{t}\right)_{t>0} \subseteq \mathcal{Y}$, where $\mathcal{Y}$ is a metric space, we consider the cluster set at $0, \mathcal{C}_{0}(Y(\omega))$, as the collection of all accumulation points of $\left(Y_{t}(\omega)\right)_{t>0}$ as $t \downarrow 0$, that is,

$$
\mathcal{C}_{0}(Y(\omega))=\bigcap_{t>0} \overline{\left\{Y_{t^{\prime}}(\omega): t^{\prime} \leq t\right\}}
$$

with the closure taken in $\mathcal{Y}$.

In the context of the family $Z=\left(Z_{t}\right)_{t>0}$ in (2.3) we only consider the metric space $\left(\mathcal{M}, d_{\mathcal{M}}\right)$ throughout. Notations like $\mathcal{C}_{0}(Z)$, and also assertions regarding the compactness of $Z$, are with reference to this space.

Finally, define

$$
\mathcal{K}=\left\{f \in \mathcal{M}: \int_{0}^{\infty} \frac{\mathrm{d} z}{f^{2}(z)} \leq 1\right\}
$$

$\mathcal{K}$ is the relevant cluster set for Wichura's result, and it plays a similar role for us. Our main result is [cf. Sect. 4 for a proof]:

Theorem 2.1 Let $T>0$. Assume $\sigma^{2}>0$ in (2.2). Then $\left(Z_{t}\right)_{0<t \leq T}$ is a.s. relatively compact and

$$
\mathcal{C}_{0}(Z)=\frac{\sigma \pi}{\sqrt{8}} \mathcal{K}, \quad \text { a.s. }
$$

Remark 2.1 We are still far from a general formulation of a result for Lévy processes at small times, that would allow us to cover the cases where $\sigma^{2}=0$. For instance, one could ask the following question: suppose that $X$ is in the domain of attraction of the normal distribution for $t \downarrow 0$. Can we find necessary and sufficient conditions, formulated in terms of the canonical triplet $\left(\gamma, \sigma^{2}, \Pi\right)$, such that, for some centering and scaling functions $b$ and $c>0$,

$$
\mathcal{C}_{0}\left(\left(\frac{\|X-b\|_{\cdot t}}{c(t)}\right)_{t>0}\right)=\mathcal{K}
$$

or, less ambitiously,

$$
\mathcal{C}_{0}\left(\left(\frac{\|X-b\|_{t}}{c(t)}\right)_{t>0}\right)=[1, \infty] ?
$$

A similar question can be asked when $X$ is not in the domain of attraction of the normal distribution, but then we expect the cluster sets in the functional version to be quite different from $\mathcal{K}$. This question is partially settled for large times by the results 
of [7] and [29]. The relevant cluster set (up to a constant multiple) in these papers is a nice generalisation of $\mathcal{K}$ in which the " 2 " is replaced by " $\alpha$ ".

We refer to Remark 4.1 at the end of Sect. 4 for further comments.

\section{Applications}

Almost sure cluster sets of a family of random elements are transmitted via continuous mappings, provided the family obeys an additional compactness property (cf. [6]). This is the analog of a continuous mapping theorem for cluster sets. In our situation, this reads as follows. Let $\mathcal{Y}$ be a metric space and $f: \mathcal{M} \rightarrow \mathcal{Y}$ a continuous mapping. Assume that $M=\left(M_{t}\right)_{t>0}$ is a family of mappings $M_{t}: \Omega \rightarrow \mathcal{M}$. Suppose that $\left(M_{t}\right)_{0<t \leq T}$ is a.s. relatively compact for some $T>0$ with cluster set $\mathcal{C}_{0}(M)$ in $\mathcal{M}$. Then both $\mathcal{C}_{0}(M)$ and $f \circ \mathcal{C}_{0}(M)$ are a.s. compact. Consider $Y=f \circ M=\left(f\left(M_{t}\right)\right)_{t>0}$ as a family of random elements in $\mathcal{Y}$. Then $\left(Y_{t}\right)_{0<t \leq T}$ is a.s. compact with cluster set $\mathcal{C}_{0}(Y)=\mathcal{C}_{0}(f \circ M)=f \circ \mathcal{C}_{0}(M)$.

Together with the continuous mapping theorem there are many potential applications of Theorem 2.1; for instance, to weighted occupation measures and first exit times, much in the spirit of Chen, Kuelbs and Li [7]. In this section we illustrate these methods by giving just two applications; others can be developed as in [7].

One-dimensional Chung law. Note that the point evaluations $f \mapsto f(t)$ are discontinuous as mappings from $\mathcal{M}$, endowed with the metric $d_{\mathcal{M}}$. Consequently, we cannot apply the continuous mapping theorem to get one-dimensional results directly from Theorem 2.1. They do follow by a slightly modified argument, however, from Theorem 2.1. This was demonstrated by [7] in a large time situation, and their arguments readily carry over to our situation. Consequently, without proof, we note the following implication of Theorem 2.1:

Corollary 3.1 Assume that $\sigma^{2}>0$ in (2.2). Then

$$
\mathcal{C}_{0}\left(\left(Z_{t}(1)\right)_{t>0}\right)=[\sigma \pi / \sqrt{8}, \infty] \text { a.s. }
$$

In particular, we have the following analogue of (1.1): assume that $\sigma^{2}>0$ in (2.2). Then for $P$-almost all $\omega \in \Omega$,

$$
\liminf _{t \downarrow 0} \frac{\sup _{0<s \leq t}|X(s, \omega)|}{\sqrt{t / \ell_{2}(t)}}=\frac{\sigma \pi}{\sqrt{8}} .
$$

Remark 3.1 Further to our discussion in Sect. 1, we expand a little on the analogy between "small time" $(t \downarrow 0)$ and "large time" $(t \uparrow \infty)$ behaviour of the Lévy process. There is often a close correspondence between them. In the present context, we can draw an analogy between random walks and Lévy processes with finite variance, for which we have (1.1), and Lévy processes with nonzero Gaussian component, $\sigma^{2}>0$, for which we have (2.6) and (3.1). Just as Chung's "other law" was first formulated for random walks with finite variance, then generalised in large time results such as those of [29] for $\alpha$-stable laws, similarly we can ask for corresponding small time 
results to ours when $\sigma^{2}=0$. So far these have not been worked out. The small time results in the case $\sigma^{2}>0$ arise, intuitively, because the Gaussian component of the process dominates the small jump component (the large jump component of course plays no part in the small time behaviour; see Lemma 4.1 below). This suggests that there might be a more direct proof of Theorem 2.1 taking advantage of this intuition, but so far we have not been able to find such a proof. There is certainly scope for further interesting research in this direction.

As a caution, however, we mention that an exact analogy between small and large time results cannot always be drawn. For example, there is no "large time" analogue of the small time square root-norm law of the iterated logarithm in Theorem 2.2 of [4] or of Theorem 2.1 of Savov [32].

Two-sided level crossings. For $u>0$ let $\tau(u)$ be the first instant that $X$ exits the region $\{y \in \mathbb{R}:|y| \leq u\}:$

$$
\tau(u)=\inf \{t>0:|X(t)|>u\}=\inf \left\{t>0:\|X\|_{t}>u\right\}, \quad u>0 .
$$

Here we employ the usual convention inf $\emptyset=\infty$. We set $\tau(0)=0$ and consider the family $U=\left(U_{t}\right)_{t>0}$ of interpolated first exit times:

$$
U_{t}=U_{t}(\cdot)=\frac{\tau(t \cdot)}{t^{2} \ell_{2}(t)}, \quad t>0
$$

Let $D_{0}$ be the space of functions $g:[0, \infty) \rightarrow[0, \infty)$ such that $g(0)=0, g$ is nondecreasing on $[0, \infty)$ and right continuous on $(0, \infty)$. By the same constructions as in (2.4), it is possible to define a metric $d_{0}$ in $D_{0}$ via the Lévy metric such that $g_{n} \rightarrow g$ in $\left(D_{0}, d_{0}\right)$, if and only if $g_{n}(s) \rightarrow g(s)$ for all continuity points $s$ of $g$ (both as $n \rightarrow \infty)$.

In the sequel, we interpret exclusively $U=\left(U_{t}\right)$ as a family of random variables in the metric space $\left(D_{0}, d_{0}\right)$. To apply the continuous mapping theorem, we introduce the mapping $\mathcal{T}: \mathcal{M} \rightarrow D_{0}$ where, for all $f \in \mathcal{M}$, function $g=\mathcal{T}(f) \in D_{0}$ is defined by setting $g(0)=0$ and

$$
g(u)=\inf \{t: f(t)>u\}, \quad u>0 .
$$

More precisely, this defines a continuous bijection $\mathcal{T}$ from $\left(\mathcal{M}, d_{\mathcal{M}}\right)$ onto $\left(D_{0}, d_{0}\right)$. Also, define

$$
\widetilde{\mathcal{K}}=\left\{g \in D_{0}: \int_{0}^{\infty} \frac{\mathrm{d} g(z)}{z^{2}} \leq 1\right\} .
$$

Note that $\mathcal{T} \circ(\alpha \mathcal{K})=(1 / \alpha)^{2} \widetilde{\mathcal{K}}$ for all $\alpha>0$, and observe that

$$
\mathcal{C}_{0}(U)=\mathcal{C}_{0}\left(\left\{u \mapsto \tau\left[u \sqrt{t / \ell_{2}(t)}\right] / t\right\}_{t>0}\right)=\mathcal{C}_{0}(\mathcal{T} \circ Z)=\mathcal{T} \circ \mathcal{C}_{0}(Z)
$$


Thus from Theorem 2.1 and the continuous mapping theorem for cluster sets, we get (the topological references are with respect to $\left.\left(D_{0}, d_{0}\right)\right)$ :

Corollary 3.2 Let $T>0$ and assume $\sigma^{2}>0$ in (2.2). Then $\left(U_{t}\right)_{0<t \leq T}$ is a.s. relatively compact and

$$
\mathcal{C}_{0}(U)=\frac{8}{\sigma^{2} \pi^{2}} \widetilde{\mathcal{K}} \text { a.s. }
$$

\section{Preliminaries to the proofs}

As foreshadowed in Sect. 1, the proof of Theorem 2.1 proceeds by approximating the given Lévy process $X$, having nonzero Gaussian component, with a Brownian motion, in analogy with modern proofs of laws of the iterated logarithm and generalised laws of the iterated logarithm in large time (finite and infinite variance) settings.

In this section we prove some auxiliary results needed in Sect. 5. We have to consider the probability of the event that $\|X\|$. visits the vicinity of certain functions $f \in \mathcal{M}$ with respect to the metric $d_{\mathcal{M}}$. Equivalently, we may study the probability that $\|X\|$. visits certain elements of a convenient basis of the topology in $\mathcal{M}$, namely:

$$
P\left(\|X\| . \in\left\{f \in \mathcal{M}: a_{k}<f\left(t_{k}\right) \leq b_{k}, 1 \leq k \leq m\right\}\right),
$$

for suitable sequences $\left\{a_{k}\right\}_{k=1}^{m}$ and $\left\{b_{k}\right\}_{k=1}^{m}$ and time points $\left\{t_{k}\right\}_{k=1}^{m} \subseteq[0, \infty)$.

Removing the large jumps from $X$ via Lemma 4.1 below, and adjusting the variance to match an approximating Brownian motion, we are left with a Lévy process $Y^{(\tau)}$, for which we can derive upper and lower bounds for probabilities such as (4.1) by normal approximation (see Propositions 4.1 and 4.2).

To this end, many arguments from [7] are useful, but we have to accommodate other features such as the lack of selfsimilarity of $X$. In Lemma 4.2 we provide a BerryEsséen type inequality for $Y^{(\tau)}$ in terms of Brownian motion. Our upper bound then follows from Lemma 4.2 and Anderson's inequality [1]. In Lemma 4.3 we give a bound on the total variation norm of Wiener measures under linear shifts. The required lower bound then follows from Lemmas 4.2 and 4.3. To make efficient use of our lower bound we have to carry out some further analysis in Lemma 4.4 where we exploit the connection to "small ball" probabilities. (See Kuelbs and Li [17], Li [19], Li and Linde [20], and Li and Shao [21]. The latter has a good overview of small ball research up to 2001, including applications to Chung's other law. See also [2,23,33-35].) At the end of this section we supply some further technical comments relevant to Theorem 2.1 in Remark 4.1.

We will make use of a "truncated" and "centered" decomposition of $X$. Define a function $v:(0, \infty) \rightarrow \mathbb{R}$ as follows:

$$
v(\tau)= \begin{cases}\gamma-\int_{\tau<|x| \leq 1} x \Pi(\mathrm{d} x), & \text { if } 0<\tau \leq 1, \\ \gamma+\int_{1<|x| \leq \tau} x \Pi(\mathrm{d} x), & \text { if } \tau>1\end{cases}
$$


This will play the role of a centering function. Throughout, the "tail sum" $\bar{\Pi}(\tau)$ is defined by

$$
\bar{\Pi}(\tau)=\Pi(\mathbb{R} \backslash[-\tau, \tau]), \quad \tau>0
$$

Define functions $V(\tau), \rho(\tau)$ and $K(\tau)$ for $\tau>0$ by

$$
V(\tau)=\sigma^{2}+\int_{0<|y| \leq \tau} y^{2} \Pi(\mathrm{d} y), \quad \rho(\tau)=\int_{0<|y| \leq \tau}|y|^{3} \Pi(\mathrm{d} y),
$$

and

$$
K(\tau)=\frac{v(\tau)}{\sqrt{V(\tau)}}
$$

where we employ the convention $0 / 0=0$ in the definition of $K$. Note that $V(\tau)$, $\rho(\tau)$, and $K(\tau)$ are finite for each $\tau>0$ by virtue of (2.1).

Throughout, let id $: \mathbb{R} \rightarrow \mathbb{R}$ be the identity function and $B=(B(t))_{t \geq 0}$ be standard Brownian motion.

For any $\tau>0$ we have the decomposition of $X$ into

$$
X=v(\tau) \mathrm{id}+X^{(\tau)}+W^{(\tau)}
$$

where $X^{(\tau)}$ and $W^{(\tau)}$ are independent Lévy processes with

$$
W^{(\tau)}(t)=\sum_{0<s \leq t} \Delta X(s) \mathbf{1}_{\{|\Delta X(s)|>\tau\}}, \quad t \geq 0 .
$$

If $V(\tau)=0$ then we set $B^{(\tau)}=Y^{(\tau)} \equiv 0$; otherwise, if $V(\tau)>0$, then we set

$$
B^{(\tau)}=B+K(\tau) \text { id } \quad \text { and } \quad Y^{(\tau)}=\frac{X^{(\tau)}}{\sqrt{V(\tau)}}+K(\tau) \mathrm{id}=\frac{X^{(\tau)}+v(\tau) \mathrm{id}}{\sqrt{V(\tau)}}
$$

Note that $E\left[X^{(\tau)}(t)\right]=0$ and $E\left[X^{(\tau)}(t)\right]^{2}=t V(\tau)$. Further, jumps are smaller than or equal in magnitude to $\tau$ for both $X^{(\tau)}$ and $Y^{(\tau)}$, and bigger in magnitude than $\tau$ for $W^{(\tau)}$. Also, we have $E\left[B^{(\tau)}(t)\right]=E\left[Y^{(\tau)}(t)\right]$ and $\operatorname{var}\left(B^{(\tau)}(t)\right)=\operatorname{var}\left(Y^{(\tau)}(t)\right)=t$ for all $t \geq 0$.

The large jumps are asymptotically negligible along suitable truncation sequences $\tau_{n} \downarrow 0$ :

Lemma 4.1 Fix $s>0$ and let $\tau_{n} \downarrow 0$ be such that $\sum_{n \geq 1} \tau_{n} \bar{\Pi}\left(\sqrt{\tau_{n}}\right)<\infty$. Then we have

$$
P\left(\left\|W^{\left(\sqrt{\tau_{n}}\right)}\right\|_{s \tau_{n}}>0 \text { i.o. as } n \rightarrow \infty\right)=0
$$


Proof of Lemma 4.1 Note that

$$
\begin{aligned}
& P\left(\left\|W^{\left(\sqrt{\tau_{n}}\right)}\right\|_{s \tau_{n}}>0\right) \\
& \quad \leq P\left(X \text { has at least one jump up to time } s \tau_{n} \text { exceeding } \sqrt{\tau_{n}} \text { in magnitude }\right) \\
& \quad=1-e^{-s \tau_{n} \bar{\Pi}\left(\sqrt{\tau_{n}}\right)} \\
& \quad \leq s \tau_{n} \bar{\Pi}\left(\sqrt{\tau_{n}}\right), \quad n \in \mathbb{N} .
\end{aligned}
$$

(Here $\mathbb{N}=\{1,2,3, \ldots\}$.) By our assumption, this is summable over $n \in \mathbb{N}$, so (4.6) holds by the Borel-Cantelli lemma.

Next we develop Brownian motion approximations for $X^{(\tau)}$ and $Y^{(\tau)}$, using a result of [4] to transfer an inequality of Sakhanenko [31] from discrete to continuous time. At this stage we do not restrict to $\sigma^{2}>0$. An important point to note is that, while $X^{(\tau)}$ is centered at $0, Y^{(\tau)}$ and $B^{(\tau)}$ are not, in Lemma 4.2. This allows us to accomodate the uncentered process $Z$ in (2.3). (Cf. also Lemma 4.3 and the use of Anderson's inequality in Proposition 4.1.)

Lemma 4.2 Set $\tau>0$, and assume that the function $V$ in (4.3) satisfies $V(\tau)>0$. Then we have:

(i) Suppose functions $g_{i}(t), t \geq 0, i=1,2$, satisfy

$$
g_{2}(t)<g_{1}(t), \quad t \geq 0, \quad \text { and } g_{2}(0)<0<g_{1}(0),
$$

and

$$
\left|g_{i}(t+h)-g_{i}(t)\right| \leq G h, \quad i=1,2, h>0, t \geq 0,
$$

for some constant $G>0$. Then for any $t>0$, we have the bound

$$
\begin{aligned}
& \mid P\left(g_{2}(s) \leq \frac{X^{(\tau)}(s)}{\sqrt{V(\tau)}} \leq g_{1}(s), 0 \leq s \leq t\right) \\
& -P\left(g_{2}(s) \leq B(s) \leq g_{1}(s), 0 \leq s \leq t\right) \mid \\
& \quad \leq \frac{C_{S}(1+G \sqrt{t}) \rho(\tau)}{\sqrt{t}(V(\tau))^{3 / 2}},
\end{aligned}
$$

where $C_{S}$ is an absolute constant and $\rho$ is defined in (4.3).

(ii) Suppose $g(t), t \geq 0$, satisfies $g(0)>0$ and

$$
|g(t+h)-g(t)| \leq G h, \quad h>0, t \geq 0,
$$


for some constant $G>0$. Then for any $t>0$, we have the bound

$$
\begin{aligned}
& \left|P\left(\left|Y^{(\tau)}(s)\right| \leq g(s), 0 \leq s \leq t\right)-P\left(\left|B^{(\tau)}(s)\right| \leq g(s), 0 \leq s \leq t\right)\right| \\
& \quad \leq \frac{C_{S}(1+\sqrt{t}[G+|K(\tau)|]) \rho(\tau)}{\sqrt{t}(V(\tau))^{3 / 2}}
\end{aligned}
$$

where $C_{S}$ is the absolute constant in (4.8).

Proof of Lemma 4.2 Observe that (ii) is implied by (i). To show (i) fix $\tau, t>0$, and $g_{1}, g_{2}$, as specified, and assume (4.7). The functions $g_{1}$ and $g_{2}$ are then continuous, and as probability measures are continuous from above, we have

$$
\begin{aligned}
& P\left(g_{2}(s) \leq \frac{X^{(\tau)}(s)}{\sqrt{V(\tau)}} \leq g_{1}(s), 0 \leq s \leq t\right) \\
& =P\left(g_{2}(t s) \leq \frac{X^{(\tau)}(t s)}{\sqrt{V(\tau)}} \leq g_{1}(t s), 0 \leq s \leq 1\right) \\
& =\lim _{n \rightarrow \infty} P\left(g_{2}\left(t j 2^{-n}\right) \leq \frac{X^{(\tau)}\left(t j 2^{-n}\right)}{\sqrt{V(\tau)}} \leq g_{1}\left(t j 2^{-n}\right), 1 \leq j \leq 2^{n}\right) \\
& =\lim _{n \rightarrow \infty} P\left(\frac{g_{2}\left(t j 2^{-n}\right)}{\sqrt{t}} \leq \frac{\sum_{m=1}^{j} \Delta_{m}^{(\tau, t)}(n)}{2^{n / 2}} \leq \frac{g_{1}\left(t j 2^{-n}\right)}{\sqrt{t}}, \quad 1 \leq j \leq 2^{n}\right)
\end{aligned}
$$

where, for $m \in \mathbb{N}=\{1,2, \ldots\}$, we set

$$
\Delta_{m}^{(\tau, t)}(n)=2^{n / 2}(t V(\tau))^{-1 / 2}\left[X^{(\tau)}\left(t m 2^{-n}\right)-X^{(\tau)}\left(t(m-1) 2^{-n}\right)\right] .
$$

For each $n \in \mathbb{N},\left(\Delta_{m}^{(\tau, t)}(n)\right)_{m \in \mathbb{N}}$ is a sequence of independent and identically distributed random variables having the same distribution as $2^{n / 2}(t V(\tau))^{-1 / 2} X^{(\tau)}\left(t 2^{-n}\right)$, thus, having zero mean and variance one. By the inequality following Eq. (4) of Sakhanenko [31], for a Brownian motion $(B(t))_{t \geq 0}$ we have that

$$
\begin{aligned}
& \mid P\left(\frac{g_{2}\left(t j 2^{-n}\right)}{\sqrt{t}} \leq \frac{\sum_{m=1}^{j} \Delta_{m}^{(\tau, t)}(n)}{2^{n / 2}} \leq \frac{g_{1}\left(t j 2^{-n}\right)}{\sqrt{t}}, 1 \leq j \leq 2^{n}\right) \\
& \quad-P\left(\frac{g_{2}(t s)}{\sqrt{t}} \leq B(s) \leq \frac{g_{1}(t s)}{\sqrt{t}}, 0 \leq s \leq 1\right) \mid \\
& \quad \leq C^{\prime} 2^{-n / 2}(1+G \sqrt{t}) E\left|\Delta_{1}^{(\tau, t)}(n)\right|^{3} \\
& \quad=\frac{C^{\prime}(1+G \sqrt{t})}{\sqrt{t}(V(\tau))^{3 / 2}} \frac{E\left|X^{(\tau)}\left(t 2^{-n}\right)\right|^{3}}{t 2^{-n}}, \quad n=1,2, \ldots,
\end{aligned}
$$


where $C^{\prime}$ is an absolute constant. By (4.9), the lefthand side of (4.10) tends as $n \rightarrow \infty$ to the lefthand side of (4.8). For the righthand side, we note that $X^{(\tau)}=\sigma B^{\prime}+\widehat{X}^{(\tau)}$, where $B^{\prime}$ is a standard Brownian motion, $\sigma \geq 0$, and

$$
\widehat{X}^{(\tau)}(u)=\text { a.s. } \lim _{\varepsilon \downarrow 0}\left(\sum_{0<s \leq u} \Delta X(s) \mathbf{1}_{\{\varepsilon<|\Delta X(s)| \leq \tau\}}-u \int_{\varepsilon<|x| \leq \tau} x \Pi(\mathrm{d} x)\right)
$$

is such that, for $u>0$,

$$
E\left|X^{(\tau)}(u)\right|^{3}=E\left|\sigma B^{\prime}(u)+\widehat{X}^{(\tau)}(u)\right|^{3} \leq 8 \sigma^{3} E\left|B^{\prime}(u)\right|^{3}+8 E\left|\widehat{X}^{(\tau)}(u)\right|^{3} .
$$

We have $u^{-1} E\left|B^{\prime}(u)\right|^{3}=u^{1 / 2} E\left|B^{\prime}(1)\right|^{3} \rightarrow 0$, and $u^{-1} E\left|\widehat{X}^{(\tau)}(u)\right|^{3} \rightarrow \rho(\tau)$ as $u \rightarrow 0$, the latter by Lemma 4.3(i) of [4]. Thus letting $n \rightarrow \infty$ in the righthand side of (4.10) gives (4.8) for the choice $C_{S}=8 C^{\prime}$.

Now we are in the position to provide our upper bound for the proof of Theorem 2.1:

Proposition 4.1 Let $\tau>0$ be such that $V(\tau)>0$. Let $m \geq 2$ and $0=t_{0}<t_{1}$ $<\cdots<t_{m}$ and $0<b_{1} \leq b_{2} \leq \cdots \leq b_{m}$. Set

$$
L\left(t_{1}, \ldots, t_{m-1} ; b_{1}, \ldots, b_{m}\right)=\max \left\{\frac{b_{k+1}-b_{k}}{t_{k}-t_{k-1}}: 1 \leq k \leq m-1\right\} .
$$

Then

$$
\begin{aligned}
& P\left(\left\|Y^{(\tau)}\right\|_{t_{k}} \leq b_{k}, \quad 1 \leq k \leq m\right) \\
& \leq\left(\frac{4}{\pi}\right)^{m-1} \exp \left[-\frac{\pi^{2}}{8}\left(\sum_{k=1}^{m-2} \frac{t_{k}-t_{k-1}}{b_{k+1}^{2}}+\frac{t_{m}-t_{m-2}}{b_{m}^{2}}\right)\right] \\
& \quad+\frac{C_{S} \rho(\tau)}{\sqrt{t_{m}}(V(\tau))^{3 / 2}}\left[1+\sqrt{t_{m}}\left(|K(\tau)|+L\left(t_{1}, \ldots, t_{m-1} ; b_{1}, \ldots, b_{m}\right)\right)\right],
\end{aligned}
$$

where $C_{S}$ is the universal constant in Lemma 4.2. Here we employ the convention $\sum_{k=1}^{n}=0$ for $n \leq 0$.

Proof of Proposition 4.1 Define $g(s)$ for $0 \leq s \leq t_{m}$ by: $g(0)=b_{1}$, and for $0<s \leq$ $t_{m}$,

$$
g(s)= \begin{cases}\frac{t_{k}-s}{t_{k}-t_{k-1}} b_{k}+\frac{s-t_{k-1}}{t_{k}-t_{k-1}} b_{k+1}, & s \in\left(t_{k-1}, t_{k}\right], k \in[1, m), \\ b_{m}, & s \in\left(t_{m-1}, t_{m}\right]\end{cases}
$$

Note that $g$ satisfies the conditions of Lemma 4.2 with $G=L\left(t_{1}, \ldots, t_{m-1} ; b_{1}, \ldots, b_{m}\right)$ as in (4.11). Since

$$
P\left(\left\|Y^{(\tau)}\right\|_{t_{k}} \leq b_{k}, \quad 1 \leq k \leq m\right) \leq P\left(\left|Y^{(\tau)}(s)\right| \leq g(s), \quad 0 \leq s \leq t_{m}\right),
$$


we, thus, get from Lemma 4.2 that

$$
\begin{aligned}
& P\left(\left\|Y^{(\tau)}\right\|_{t_{k}} \leq b_{k}, 1 \leq k \leq m\right) \\
& \leq P\left(\left|B^{(\tau)}(s)\right| \leq g(s), \quad 0 \leq s \leq t_{m}\right) \\
& +\frac{C_{S} \rho(\tau)}{\sqrt{t_{m}}(V(\tau))^{3 / 2}}\left[1+\sqrt{t_{m}}(|K(\tau)|+G)\right] .
\end{aligned}
$$

On the other hand, we have

$$
\begin{aligned}
& P\left(\left|B^{(\tau)}(s)\right| \leq g(s), \quad 0 \leq s \leq t_{m}\right) \\
& \quad \leq P\left(\left\|B^{(\tau)}\right\|_{t_{k}} \leq b_{k+1}, \quad 1 \leq k \leq m-2, \quad\left\|B^{(\tau)}\right\|_{t_{m}} \leq b_{m}\right),
\end{aligned}
$$

and, thus, by Anderson's inequality (cf. Anderson [1]),

$$
\begin{aligned}
& P\left(\left|B^{(\tau)}(s)\right| \leq g(s), \quad 0 \leq s \leq t_{m}\right) \\
& \quad \leq P\left(\|B\|_{t_{k}} \leq b_{k+1}, \quad 1 \leq k \leq m-2, \quad\|B\|_{t_{m}} \leq b_{m}\right) .
\end{aligned}
$$

Using the same argument as in [7] (cf. p.264) to bound the righthand side of (4.13), we can conclude that

$$
\begin{aligned}
& P\left(\left|B^{(\tau)}(s)\right| \leq g(s), \quad 0 \leq s \leq t_{m}\right) \\
& \quad \leq \prod_{k=1}^{m-2} P\left(\|B\|_{t_{k}-t_{k-1}} \leq b_{k+1}\right) P\left(\|B\|_{t_{m}-t_{m-2}} \leq b_{m}\right) .
\end{aligned}
$$

Recall that (cf. Chung [8], p.221, or Jain and Pruitt [14])

$$
P\left(\|B\|_{1} \leq x\right) \leq \frac{4}{\pi} \exp \left(-\frac{\pi^{2}}{8 x^{2}}\right), \quad x>0,
$$

and, thus, by the selfsimilarity of Brownian motion and (4.14),

$$
\begin{aligned}
& P\left(\left|B^{(\tau)}(s)\right| \leq g(s), \quad 0 \leq s \leq t_{m}\right) \\
& \quad \leq\left(\frac{4}{\pi}\right)^{m-1} \exp \left[-\frac{\pi^{2}}{8}\left(\sum_{k=1}^{m-2} \frac{t_{k}-t_{k-1}}{b_{k+1}^{2}}+\frac{t_{m}-t_{m-2}}{b_{m}^{2}}\right)\right] .
\end{aligned}
$$

The proof is completed by combining (4.12) and (4.15).

For the lower bound, we use Girsanov's theorem, in the next lemma, to deal with the centering issue. For $t>0$ let $\mathcal{C}[0, t]$ be the Borel field in the space of continuous 
functions $f:[0, t] \rightarrow \mathbb{R}$, endowed with the uniform topology. Then we have the following fact concerning Brownian motion:

Lemma 4.3 There exists a universal constant $C_{B} \in(0, \infty)$ such that

$$
\sup _{F \in \mathcal{C}[0, t]} \mid P(B \in F)-P(B+x \text { id } \in F)\left|\leq C_{B} \sqrt{t}\right| x \mid,
$$

for all $x \in \mathbb{R}$ and $t>0$ with $\sqrt{t}|x| \leq 1$.

Proof of Lemma 4.3 Let $C_{B}=E\left[(|B(1)|+1) e^{|B(1)|+1}\right]$. Let $t>0$ and $x \in \mathbb{R}$ such that $\sqrt{t}|x| \leq 1$. It follows from Girsanov's theorem, e.g., Theorem 3.5.1 in [15], that

$$
P(B+x \text { id } \in F)=E\left[\mathbf{1}_{B \in F} \exp \left(x B(t)-\frac{x^{2}}{2} t\right)\right], \quad F \in \mathcal{C}([0, t]),
$$

and thus by the elementary inequality $\left|e^{y}-1\right| \leq|y| e^{|y|}(y \in \mathbb{R})$,

$$
|P(B \in F)-P(B+x \mathrm{id} \in F)| \leq E\left|\exp \left(x B(t)-\frac{x^{2}}{2} t\right)-1\right| \leq C_{B} \sqrt{t}|x|,
$$

for all $F \in \mathcal{C}([0, t])$, as required.

Now we can provide the lower bound for our proof of Theorem 2.1. It is convenient to introduce the function, for $0<c<a<b$ and $0<T<1$,

$$
\Theta(a, b, c, T)=P\left(\|B\|_{1}>a,\|B\|_{T} \leq b, \sup _{T \leq s \leq 1}|B(s)| \leq c\right) .
$$

Proposition 4.2 Let $m \geq 1$ and $0=t_{0}<t_{1}<\cdots<t_{m}$ and set $\Delta t_{k}=t_{k}-t_{k-1}$ for $1 \leq k \leq m$. Let $0<T<1$. Let $\left\{a_{k}\right\}_{k=0}^{m}$ and $\left\{b_{k}\right\}_{k=0}^{m}$ be sequences satisfying

$$
0=a_{0}=b_{0}<a_{1}<b_{1} \leq a_{2}<b_{2} \leq \cdots \leq a_{m}<b_{m}
$$

Let $0<\delta<\min \left\{a_{1}, 1\right\}$ such that

$$
\delta\left(b_{k}-b_{k-1}\right)<(1+\delta) a_{k}<(1-\delta) b_{k}, \quad 1 \leq k \leq m .
$$

Let $\tau>0$ and suppose that $V(\tau)>0$ and $\sqrt{t_{m}}|K(\tau)| \leq 1$.

Set

$$
\Delta t_{m}^{*}=\min \left\{\Delta t_{k}: 1 \leq k \leq m\right\} .
$$


Then

$$
\begin{aligned}
& P\left(\left\|Y^{(\tau)}\right\|_{t_{k}} \in\left(a_{k}, b_{k}\right], 1 \leq k \leq m\right) \\
& \geq \prod_{k=1}^{m} \Theta\left(\frac{(1+\delta) a_{k}}{\sqrt{\Delta t_{k}}}, \frac{(1-\delta) b_{k}}{\sqrt{\Delta t_{k}}}, \frac{\delta\left(b_{k}-b_{k-1}\right)}{\sqrt{\Delta t_{k}}}, T\right) \\
& -\frac{m C}{1-T}\left[\sqrt{t_{m}}|K(\tau)|+\frac{\rho(\tau)}{V(\tau)^{3 / 2} \sqrt{\Delta t_{m}^{*}}}\left(1+\frac{b_{m}}{\sqrt{\Delta t_{m}^{*}}}\right)\right],
\end{aligned}
$$

with $C:=4 C_{S}+C_{B}$, where $C_{S}$ and $C_{B}$ are the universal constants in Lemmas 4.2 and 4.3 , respectively.

Proof of Proposition 4.2 Let $0<T<1$ and $\alpha, \beta \in \mathbb{R}$ and define a function $g(s ; T, \alpha, \beta)$ by

$$
g(s ; T, \alpha, \beta)= \begin{cases}\alpha, & 0 \leq s \leq T \\ \frac{1-s}{1-T} \alpha+\frac{s-T}{1-T} \beta, & T<s \leq 1\end{cases}
$$

For each $1 \leq k \leq m$ let $T_{k}^{*} \in(T, 1)$ be the unique solution of

$$
a_{k}(1+\delta)=g\left(T_{k}^{*} ; T,(1-\delta) b_{k}, \delta\left(b_{k}-b_{k-1}\right)\right)
$$

With $L_{k}$ and $L_{k}^{*}$ denoting the minimal Lipschitz constants of the functions $s \mapsto$ $g\left(s / \Delta t_{k} ; T,(1-\delta) b_{k}, \delta\left(b_{k}-b_{k-1}\right)\right)$ and $s \mapsto g\left(s / \Delta t_{k} ; T_{k}^{*},(1+\delta) a_{k}, \delta\left(b_{k}-b_{k-1}\right)\right)$, respectively, it follows from our construction that

$$
L_{k}=L_{k}^{*}=\frac{(1-\delta) b_{k}-\delta\left(b_{k}-b_{k-1}\right)}{(1-T) \Delta t_{k}} \leq \frac{b_{m}}{(1-T) \Delta t_{m}^{*}}, \quad 1 \leq k \leq m,
$$

where $\Delta t_{m}^{*}$ is defined as in (4.18).

Recall (4.5). When $Q$ is any one of $Y^{(\tau)}, B$, or $B^{(\tau)}$, and $1 \leq k \leq m$, we write

$$
\begin{aligned}
p_{k}(Q):= & P\left(\left\{|Q(s)| \leq g\left(\frac{s}{\Delta t_{k}} ; T, b_{k}(1-\delta), \delta\left(b_{k}-b_{k-1}\right)\right), \quad 0 \leq s \leq \Delta t_{k}\right\}\right. \\
& \left.\cap\left\{\|Q\|_{\Delta t_{k}}>a_{k}(1+\delta)\right\}\right) \\
= & P\left(|Q(s)| \leq g\left(\frac{s}{\Delta t_{k}} ; T, b_{k}(1-\delta), \delta\left(b_{k}-b_{k-1}\right)\right), 0 \leq s \leq \Delta t_{k}\right) \\
& -P\left(|Q(s)| \leq g\left(\frac{s}{\Delta t_{k}} ; T_{k}^{*}, a_{k}(1+\delta), \delta\left(b_{k}-b_{k-1}\right)\right), 0 \leq s \leq \Delta t_{k}\right) .
\end{aligned}
$$


Recall $\sqrt{t_{m}}|K(\tau)| \leq 1$ and $0<T<1$. Next we apply the inequality in assertion (ii) of Lemma 4.2 to get from (4.19) that, for all $1 \leq k \leq m$,

$$
\begin{aligned}
& \left|p_{k}\left(Y^{(\tau)}\right)-p_{k}\left(B^{(\tau)}\right)\right| \\
& \quad \leq \frac{2 C_{S} \rho(\tau)}{\sqrt{\Delta t_{k}}(V(\tau))^{3 / 2}}\left(1+\sqrt{\Delta t_{k}}\left(|K(\tau)|+\frac{b_{m}}{(1-T) \Delta t_{m}^{*}}\right)\right) \\
& \quad \leq \frac{4 C_{S} \rho(\tau)}{(1-T)(V(\tau))^{3 / 2} \sqrt{\Delta t_{m}^{*}}}\left(1+\frac{b_{m}}{\sqrt{\Delta t_{m}^{*}}}\right) .
\end{aligned}
$$

As $\sqrt{t_{m}}|K(\tau)| \leq 1$ it follows from (4.16) that

$$
\left|p_{k}\left(B^{(\tau)}\right)-p_{k}(B)\right| \leq C_{B} \sqrt{\Delta t_{k}}|K(\tau)|
$$

for all $1 \leq k \leq m$, where $C_{B}$ is the universal constant in Lemma 4.3.

With $\bar{C}=C_{B}+4 C_{S}$, combining (4.20) and (4.21) yields

$$
\begin{aligned}
& \left|\prod_{k=1}^{m} p_{k}\left(Y^{(\tau)}\right)-\prod_{k=1}^{m} p_{k}(B)\right| \leq \sum_{k=1}^{m}\left|p_{k}\left(Y^{(\tau)}\right)-p_{k}(B)\right| \\
& \quad \leq \frac{m C}{1-T}\left[\sqrt{t_{m}}|K(\tau)|+\frac{\rho(\tau)}{(V(\tau))^{3 / 2} \sqrt{\Delta t_{m}^{*}}}\left(1+\frac{b_{m}}{\sqrt{\Delta t_{m}^{*}}}\right)\right] .
\end{aligned}
$$

For $1 \leq k \leq m$ define

$$
\begin{gathered}
A_{k}=\left\{\sup _{t_{k-1}<s \leq t_{k}}\left|Y^{(\tau)}(s)-Y^{(\tau)}\left(t_{k-1}\right)\right| \in\left(a_{k}(1+\delta), b_{k}(1-\delta)\right],\right. \\
\left.\left|Y^{(\tau)}\left(t_{k}\right)-Y^{(\tau)}\left(t_{k-1}\right)\right| \leq \delta\left(b_{k}-b_{k-1}\right)\right\} .
\end{gathered}
$$

By adapting an argument of [7] (cf. p.266), we get from the independence and stationarity of the increments of $Y^{(\tau)}$ that

$$
P\left(\left\|Y^{(\tau)}\right\|_{t_{k}} \in\left(a_{k}, b_{k}\right], 1 \leq k \leq m\right) \geq \prod_{k=1}^{m} P\left(A_{k}\right) \geq \prod_{k=1}^{m} p_{k}\left(Y^{(\tau)}\right) .
$$

Finally, it follows from the selfsimilarity of $B$ (recall (4.17)) that

$$
p_{k}(B) \geq \Theta\left(\frac{(1+\delta) a_{k}}{\sqrt{\Delta t_{k}}}, \frac{(1-\delta) b_{k}}{\sqrt{\Delta t_{k}}}, \frac{\delta\left(b_{k}-b_{k-1}\right)}{\sqrt{\Delta t_{k}}}, T\right), \quad 1 \leq k \leq m
$$

In view of (4.22)-(4.24), this completes the proof of the proposition. 
The behaviour of the function $\Theta(a, b, c)$ in Proposition 4.2 for small values of $a, b, c$ is all we really need for the proof of Theorem 2.1. To get this, we exploit a connection to the small ball probabilities of Brownian motion, in the next lemma.

Actually, without further effort, we can state a somewhat more general result than we need. Let $\alpha \in(0,2]$ and $U^{(\alpha)}=\left(U^{(\alpha)}(t)\right)_{t \geq 0}$ be a symmetric stable Lévy process of index $\alpha$. As shown by Mogul'skii [25], there exists a constant $u_{\alpha} \in(0, \infty)$ such that

$$
\lim _{\varepsilon \downarrow 0} \varepsilon^{\alpha} \log P\left(\left\|U^{(\alpha)}\right\|_{1} \leq \varepsilon\right)=-u_{\alpha} .
$$

In particular, by taking $\alpha=2$ and recalling that $U^{(2)}=B$ is a Brownian motion, we have $u_{2}=\pi^{2} / 8$.

In the next lemma we extend (4.25) to the following situation:

Lemma 4.4 Let $\alpha \in(0,2]$ and $U_{\alpha}$ be a symmetric stable Lévy process of index $\alpha$. Let $0<c<a<b$ and $0<T<1$. Then, with $u_{\alpha}$ as in (4.25), we have, as $\varepsilon \downarrow 0$,

$$
-\frac{\varepsilon^{\alpha}}{u_{\alpha}} \log P\left(\left\|U^{(\alpha)}\right\|_{1}>\varepsilon a,\left\|U^{(\alpha)}\right\|_{T} \leq \varepsilon b, \sup _{T \leq s \leq 1}\left|U^{(\alpha)}(s)\right| \leq \varepsilon c\right) \rightarrow \frac{T}{b^{\alpha}}+\frac{1-T}{c^{\alpha}}
$$

and, in particular,

$$
\lim _{\varepsilon \downarrow 0} \varepsilon^{2} \log \Theta(\varepsilon a, \varepsilon b, \varepsilon c, T)=-\frac{\pi^{2}}{8}\left(\frac{T}{b^{2}}+\frac{1-T}{c^{2}}\right),
$$

with $\Theta$ as defined in (4.17).

Proof of Lemma 4.4 Let $\alpha \in(0,2]$ and let $U=U^{(\alpha)}$ be a symmetric stable Lévy process of order $\alpha$. For all $\eta>0$ (cf. Lemma 2.3 in [7]), we have

$$
\lim _{\varepsilon \downarrow 0} \varepsilon^{\alpha} \log P\left(\|U\|_{1} \leq \varepsilon\right)=\lim _{\varepsilon \downarrow 0} \varepsilon^{\alpha} \log P\left(\|U\|_{1} \leq \varepsilon,|U(1)| \leq \varepsilon \eta\right)=-u_{\alpha} .
$$

It follows from the càdlàg property of the sample paths of $U$ and Lemma 2.1 in [7] that, for all $y \in \mathbb{R}$,

$$
P\left(\|U+y\|_{1-T} \leq \varepsilon c\right) \leq P\left(\|U\|_{1-T} \leq \varepsilon c\right) .
$$

Define

$$
A_{\varepsilon}=\left\{\|U\|_{T} \leq \varepsilon b, \sup _{T \leq s \leq 1}|U(s)| \leq \varepsilon c\right\} .
$$


We get from (4.27) that

$$
\begin{aligned}
P\left(A_{\varepsilon}\right) & =\int P\left(\|U\|_{T} \leq \varepsilon b \mid U(T)=y\right) P\left(\|U+y\|_{1-T} \leq \varepsilon c\right) P^{U(T)}(\mathrm{d} y) \\
& \leq P\left(\|U\|_{T} \leq \varepsilon b\right) P\left(\|U\|_{1-T} \leq \varepsilon c\right)
\end{aligned}
$$

Hence, by the selfsimilarity of $U$ and (4.26),

$$
\underset{\varepsilon \downarrow 0}{\lim \sup } \varepsilon^{\alpha} \log P\left(A_{\varepsilon}\right) \leq-u_{\alpha}\left(\frac{T}{b^{\alpha}}+\frac{1-T}{c^{\alpha}}\right) .
$$

Pick $0<\delta<1$ and observe that

$$
\begin{aligned}
P\left(A_{\varepsilon}\right) & \geq \int_{|y| \leq \varepsilon c \delta} P\left(\|U\|_{T} \leq \varepsilon b \mid U(T)=y\right) P\left(\|U+y\|_{1-T} \leq \varepsilon c\right) P^{U(T)}(\mathrm{d} y) \\
& \geq P\left(\|U\|_{T} \leq \varepsilon b,|U(T)| \leq \varepsilon c \delta\right) P\left(\|U\|_{1-T} \leq \varepsilon c(1-\delta)\right)
\end{aligned}
$$

and, thus, by the selfsimilarity of $U$ and (4.26),

$$
\liminf _{\varepsilon \downarrow 0} \varepsilon^{\alpha} \log P\left(A_{\varepsilon}\right) \geq-u_{\alpha}\left(\frac{T}{b^{\alpha}}+\frac{1-T}{c^{\alpha}(1-\delta)^{\alpha}}\right), \quad 0<\delta<1 .
$$

Taking $\delta \downarrow 0$ in this, and recalling (4.29), we get

$$
\lim _{\varepsilon \downarrow 0} \varepsilon^{\alpha} \log P\left(A_{\varepsilon}\right)=-u_{\alpha}\left(\frac{T}{b^{\alpha}}+\frac{1-T}{c^{\alpha}}\right) .
$$

By the same argument as in (4.28) we must have (recall $0<a<b$ )

$$
P\left(A_{\varepsilon} \cap\left\{\|U\|_{1} \leq \varepsilon a\right\}\right) \leq P\left(\|U\|_{T} \leq \varepsilon a\right) P\left(\|U\|_{1-T} \leq \varepsilon c\right),
$$

and, thus, by the selfsimilarity of $U$ and (4.26) and (4.30),

$$
\underset{\varepsilon \downarrow 0}{\lim \sup } \varepsilon^{\alpha} \log P\left(\|U\|_{1} \leq \varepsilon a \mid A_{\varepsilon}\right) \leq-T u_{\alpha} \frac{b^{\alpha}-a^{\alpha}}{a^{\alpha} b^{\alpha}} .
$$

In particular, we, thus, have that $P\left(\|U\|_{1} \leq \varepsilon a \mid A_{\varepsilon}\right) \rightarrow 0$ as $\varepsilon \downarrow 0$. Note that

$$
\begin{gathered}
\varepsilon^{\alpha} \log P\left(\|U\|_{1}>\varepsilon a,\|U\|_{T} \leq \varepsilon b, \sup _{T \leq s \leq 1}|U(s)| \leq \varepsilon c\right) \\
=\varepsilon^{\alpha} \log P\left(A_{\varepsilon}\right)+\varepsilon^{\alpha} \log \left[1-P\left(\|U\|_{1} \leq \varepsilon a \mid A_{\varepsilon}\right)\right] .
\end{gathered}
$$

This completes the proof of the lemma, in view of (4.30). 
The next lemma collects some necessary convergences. They all follow from the fact that $\int_{|x| \leq 1} x^{2} \Pi(\mathrm{d} x)<\infty$, for any Lévy canonical measure, so we have omitted the proofs (however, cf. [4] for the case $\tau_{n}=r^{n}$ ).

Lemma 4.5 Suppose $\left(\tau_{n}\right)_{n=1,2, \ldots}$ is either the sequence $r^{n}$, where $0<r<1$, or the sequence $n^{-n}$. Then we have

$$
\sum_{n \geq 1} \tau_{n} \bar{\Pi}\left(\tau_{n}^{1 / 2}\right)+\sum_{n \geq 1} \tau_{n}^{-1 / 2} \rho\left(\tau_{n}^{1 / 2}\right)+\sum_{n \geq 1} \tau_{n}^{1 / 2}\left|\nu\left(\tau_{n}^{1 / 2}\right)\right|<\infty
$$

Finally, in this section, for convenience we summarize some properties of $\mathcal{K}$ into a lemma. Its proof is postponed to an Appendix.

Lemma 4.6 Let $\mathcal{K}$ be as in (2.5). Then:

(i) $\mathcal{K}$ is a closed and compact subset of $\left(\mathcal{M}, d_{\mathcal{M}}\right)$.

(ii) Let $f \in \mathcal{M} \backslash \mathcal{K}$. Then there exist $\delta>0$ and $m \geq 2$, together with $0=s_{0}<s_{1}$ $<\ldots<s_{m}$, such that $f\left(s_{m}\right)<\infty$ and

$$
I_{\delta}^{m}(f):=\sum_{k=1}^{m-2} \frac{s_{k}-s_{k-1}}{\left(f\left(s_{k+1}\right)+\delta\right)^{2}}+\frac{s_{m}-s_{m-2}}{\left(f\left(s_{m}\right)+\delta\right)^{2}}>1 .
$$

(iii) Let $f \in \mathcal{K}$ and $\varepsilon>0$. Then there exist $m \geq 1$ and $0=s_{0}<s_{1}<\cdots<s_{m}$ and $0<a_{1}<b_{1} \leq a_{2}<b_{2} \leq \cdots \leq a_{m}<b_{m}$ such that $\sum_{k=1}^{m} \frac{s_{k}-s_{k-1}}{b_{k}^{2}}<1$ and $\left\{g \in \mathcal{M}: a_{k}<g\left(s_{k}\right) \leq b_{k}, 1 \leq k \leq m\right\} \subseteq B_{\varepsilon}(f):=\{g \in \mathcal{M}:$ $\left.d_{\mathcal{M}}(g, f)<\varepsilon\right\}$.

Remark 4.1 Note that, so far in the present section, we have not imposed the restriction $\sigma^{2}>0$, only the weaker requirement that

$$
V(\tau)>0, \text { for all } \tau>0 .
$$

This is a natural condition since if $V(\tau)=0$ for some $\tau>0$ then $X$ degenerates to a compound Poisson process with drift, and local behaviour is not an issue. In Theorem 2.1, however, and from now on, we assume the stronger condition $\sigma^{2}>0$. As discussed in Remark 3.1, it is unclear to us at the present stage how to generalise Theorem 2.1. It might be fruitful, as suggested by a referee, to try to analyse a subordinated Brownian motion along these lines (cf. also [22]).

\section{Proof of Theorem 2.1}

We first show that $\mathcal{C}_{0}(Z)$ is a subset of a particular multiple of $\mathcal{K}$. Recall that $Z$ is defined in (2.3).

Lemma 5.1 Assume $\sigma^{2}>0$ in (2.2). Then

$$
\mathcal{C}_{0}(Z) \subseteq \frac{\sigma \pi}{\sqrt{8}} \mathcal{K}, \quad \text { a.s. }
$$


Proof of Lemma 5.1 Let $f \in \mathcal{M} \backslash\left(\frac{\sigma \pi}{\sqrt{8}} \mathcal{K}\right)$. In view of Lemma 4.6(ii), we can find $\delta>0, m \geq 2$, and $0=s_{0}<s_{1}<\ldots<s_{m}$, such that $f\left(s_{m}\right)<\infty$ and

$$
I_{\delta}^{m}(f)>\frac{8}{\sigma^{2} \pi^{2}}
$$

Fix this $\delta$ and $m$ throughout the proof. For $0<\delta^{\prime} \leq \delta$ define

$$
N_{\delta^{\prime}}^{m}(f)=\left\{g \in \mathcal{M}: g\left(s_{k}\right)<f\left(s_{k}\right)+\delta^{\prime}, \quad 1 \leq k \leq m\right\}
$$

Fix $0<r<1$. For $n=1,2, \ldots$ let $\tau_{n}=r^{n}$. Let $C_{S}$ be the constant in Lemma 4.2 and set

$$
L:=\max \left\{\frac{f\left(s_{k+1}\right)-f\left(s_{k}\right)}{s_{k}-s_{k-1}}: 1 \leq k \leq m-1\right\} .
$$

Since $V(\tau) \geq \sigma^{2}>0$ for all $\tau>0$, we get from Proposition 4.1 with $\tau$ replaced by $\sqrt{\tau_{n}}, t_{k}=s_{k} \tau_{n}$, and $b_{k}:=\sqrt{\tau_{n}}\left(f\left(s_{k}\right)+\delta\right) / \sqrt{V\left(\sqrt{\tau_{n}}\right) \ell_{2}\left(\tau_{n}\right)}$, that

$$
\begin{aligned}
P\left(\frac{\left\|X^{\left(\sqrt{\tau_{n}}\right)}+v\left(\sqrt{\tau_{n}}\right) \mathrm{id}\right\|_{\tau_{n}}}{\sqrt{\tau_{n} / \ell_{2}\left(\tau_{n}\right)}} \in N_{\delta}^{m}(f)\right) \\
=P\left(\left\|Y^{\left(\sqrt{\tau_{n}}\right)}\right\|_{\tau_{n}} \in \sqrt{\frac{\tau_{n}}{V\left(\sqrt{\tau_{n}}\right) \ell_{2}\left(\tau_{n}\right)}} N_{\delta}^{m}(f)\right) \\
\leq\left(\frac{4}{\pi}\right)^{m-1} \exp \left(-\frac{\pi^{2}}{8} V\left(\sqrt{\tau_{n}}\right) I_{\delta}^{m}(f) \ell_{2}\left(\tau_{n}\right)\right) \\
+\frac{C_{S} \rho\left(\sqrt{\tau_{n}}\right)}{\sqrt{s_{m} \tau_{n} V\left(\sqrt{\tau_{n}}\right)^{3}}}\left[1+\sqrt{s_{m}}\left(\left|K\left(\sqrt{\tau_{n}}\right)\right|+\frac{L}{\sqrt{\tau_{n} V\left(\sqrt{\tau_{n}}\right) \ell_{2}\left(\tau_{n}\right)}}\right)\right] \\
\leq \\
\quad\left(\frac{4}{\pi}\right)^{m-1} \exp \left(-\frac{\sigma^{2} \pi^{2}}{8} I_{\delta}^{m}(f) \ell_{2}\left(\tau_{n}\right)\right) \\
\quad+\frac{C_{S} \rho\left(\sqrt{\tau_{n}}\right)}{\sigma^{4} \sqrt{s_{m} \tau_{n}}}\left(\sigma+\sqrt{s_{m} \tau_{n}}\left|v\left(\sqrt{\tau_{n}}\right)\right|+\sqrt{s_{m}} L\right)
\end{aligned}
$$

where $L$ is defined in (5.3). Recall that $\tau_{n}=r^{n}$ with $0<r<1$. By Lemma 4.5 and (5.1) we, thus, get from (5.4), for each $m=1,2, \ldots$, that

$$
\sum_{n \geq 1} P\left(\frac{\left\|X^{\left(\sqrt{\tau_{n}}\right)}+v\left(\sqrt{\tau_{n}}\right) \mathrm{id}\right\|_{\cdot \tau_{n}}}{\sqrt{\tau_{n} / \ell_{2}\left(\tau_{n}\right)}} \in N_{\delta}^{m}(f)\right)<\infty
$$


and, thus, by the Borel-Cantelli lemma,

$$
P\left(\frac{\left\|X^{\left(\sqrt{\tau_{n}}\right)}+v\left(\sqrt{\tau_{n}}\right) \mathrm{id}\right\|_{\tau_{n}}}{\sqrt{\tau_{n} / \ell_{2}\left(\tau_{n}\right)}} \in N_{\delta}^{m}(f) \text { i.o. as } n \rightarrow \infty\right)=0 .
$$

Next recall (4.4); it follows from (4.31) and Lemma 4.1 that

$$
P\left(\left\|W^{\left(\sqrt{\tau_{n}}\right)}\right\|_{s_{m} \tau_{n}}>0 \text { i.o. as } n \rightarrow \infty\right)=0 .
$$

In view of (5.6), we can replace $X^{\left(\sqrt{\tau_{n}}\right)}+v\left(\sqrt{\tau_{n}}\right)$ id with $X$ in (5.5) and get

$$
P\left(\|Z\| \cdot r^{n}=\frac{\|X\|_{\cdot r^{n}}}{\sqrt{r^{n} / \ell_{2}\left(r^{n}\right)}} \in N_{\delta}^{m}(f) \text { i.o. as } n \rightarrow \infty\right)=0, \quad 0<r<1 \text {. }
$$

Next let $\delta^{\prime} \in(0, \delta)$ and assume by way of contradiction that $P\left(\mathcal{C}_{0}(Z) \cap N_{\delta^{\prime}}^{m}(f) \neq\right.$ $\emptyset)>0$. Choose $r \in(0,1)$ and $\varepsilon>0$ such that

$$
f\left(s_{k}\right)+\delta \geq \frac{1+\varepsilon}{\sqrt{r}}\left(f\left(s_{k}\right)+\delta^{\prime}\right), \quad 1 \leq k \leq m
$$

Since $N_{\delta^{\prime}}^{m}(f)$ is open for all $0<\delta^{\prime} \leq \delta$, we can find $\widetilde{\Omega} \in \mathcal{F}$ with $P(\widetilde{\Omega})>0$ such that, for all $\omega \in \widetilde{\Omega}$, there exists a sequence $t_{n}=t_{n}(\omega) \downarrow 0$ as $n \rightarrow \infty$ with $Z_{t_{n}}(\omega) \in N_{\delta^{\prime}}^{m}(f)$ for all $n \in \mathbb{N}$. Let $\omega \in \widetilde{\Omega}$ and define a strictly increasing sequence $\left(\mu_{v}\right)=\left(\mu_{v}(\omega)\right) \subseteq \mathbb{N}$ such that $r^{\mu_{v}} \leq t_{v}<r^{\mu_{v}-1}$. Then $\mu_{v}=\mu_{v}(\omega) \uparrow \infty$ and $\ell_{2}\left(r^{\mu_{v}}\right) / \ell_{2}\left(r^{\mu_{v}-1}\right) \rightarrow 1$ as $v \rightarrow \infty$. Now (cf. (5.2)) $Z_{t_{v}}(\omega) \in N_{\delta^{\prime}}^{m}(f)$ means that $\|X\|_{s_{k} t_{v}} / \sqrt{t_{v} / \ell_{2}\left(t_{v}\right)}<f\left(s_{k}\right)+\delta^{\prime}$ for $1 \leq k \leq m$. By (5.8) there exists $v_{0}=v_{0}(\omega, \varepsilon)$ such that, for all $v \geq v_{0}$ and $1 \leq k \leq m$,

$$
f\left(s_{k}\right)+\delta \geq \frac{1+\varepsilon}{\sqrt{r}}\left(f\left(s_{k}\right)+\delta^{\prime}\right)>\frac{1}{\sqrt{r}} \sqrt{\frac{\ell_{2}\left(r^{\mu_{v}}\right)}{\ell_{2}\left(r^{\mu_{v}-1}\right)}} \frac{\|X\|_{s_{k} t_{v}}}{\sqrt{t_{v} / \ell_{2}\left(t_{v}\right)}} \geq \frac{\|X\|_{s_{k} r^{\mu_{v}}}}{\sqrt{r^{\mu_{v}} / \ell_{2}\left(r^{\mu_{v}}\right)}} .
$$

But this would contradict (5.7) since $P(\tilde{\Omega})>0$.

Consequently, we must have $\mathcal{C}_{0}(Z) \cap N_{\delta^{\prime}}^{m}(f)=\emptyset$, a.s., for all $0<\delta^{\prime}<\delta$, and, thus, $\mathcal{C}_{0}(Z) \cap N_{\delta}^{m}(f)=\emptyset$, a.s. This is because $N_{\delta}^{m}=\bigcup_{\delta^{\prime} \in(0, \delta) \cap \mathbb{Q}} N_{\delta^{\prime}}^{m}(f)$ and

$$
P\left(\mathcal{C}_{0}(Z) \cap N_{\delta}^{m}(f) \neq \emptyset\right) \leq P\left(\bigcup_{\delta^{\prime} \in(0, \delta) \cap \mathbb{Q}}\left\{\mathcal{C}_{0}(Z) \cap N_{\delta^{\prime}}^{m}(f) \neq \emptyset\right\}\right)=0 .
$$

To summarize, for all $f \in \mathcal{W}:=\mathcal{M} \backslash((\sigma \pi / \sqrt{8}) \mathcal{K})$, there exists an open neighbourhood $N(f)$ of $f$ such that $\mathcal{C}_{0}(Z) \cap N(f)=\emptyset$, a.s.

The proof is now completed along the following lines: as $\mathcal{W}$ is open by Lemma 4.6 and $\mathcal{M}$ is separable we can find a sequence $\left(f_{j}\right)_{j \in \mathbb{N}}$, dense in $\mathcal{W}$. As shown above, for 
each $j$ there is an open neighbourhood $N_{j}^{m}$ of $f_{j}$ such that $P\left(\mathcal{C}_{0}(Z) \cap N_{j}^{m}=\emptyset\right)=1$. Since $\mathcal{W} \subseteq \bigcup_{j=1}^{\infty} N_{j}^{m}$ we have

$$
\left\{\mathcal{C}_{0}(Z) \cap \mathcal{W} \neq \emptyset\right\} \subseteq\left\{\mathcal{C}_{0}(Z) \cap \bigcup_{j=1}^{\infty} N_{j}^{m} \neq \emptyset\right\} \subseteq \bigcup_{j=1}^{\infty}\left\{\mathcal{C}_{0}(Z) \cap N_{j}^{m} \neq \emptyset\right\}
$$

Consequently, $\mathcal{C}_{0}(Z) \cap \mathcal{W}=\emptyset$, a.s., and, thus, $\mathcal{C}_{0}(Z) \subseteq(\sigma \pi / \sqrt{8}) \mathcal{K}$, a.s. This completes the proof of the lemma.

Next we show the other inclusion by looking at the behaviour of $Z$ along the thin subsequence $\tau_{n}=n^{-n}$. This choice is suggested by the successful use of $n^{n}$ in the proof of the large time "other" law of the iterated logarithm of [14]. For a sequence $\left(\tau_{n}\right)_{n \in \mathbb{N}}$ of positive real numbers and $Y=\left(Y_{t}\right)_{t>0} \subseteq \mathcal{Y}$ we define

$$
\mathcal{C}_{\left(\tau_{n}\right)}(Y(\omega))=\bigcap_{n \geq 1} \overline{\left\{Y_{\tau_{m}}(\omega): m \geq n\right\}}
$$

with the closure taken in $\mathcal{Y}$.

Lemma 5.2 Assume $\sigma^{2}>0$ in (2.2). Then

$$
\frac{\sigma \pi}{\sqrt{8}} \mathcal{K} \subseteq \mathcal{C}_{\left(n^{-n}\right)}(Z) \subseteq \mathcal{C}_{0}(Z), \quad \text { a.s. }
$$

Proof of Lemma 5.2 Take $m \in \mathbb{N}$ and $0=s_{0}<s_{1}<s_{1}<\cdots<s_{m}$, and let $\left\{a_{k}\right\}_{k=0}^{m}$ and $\left\{b_{k}\right\}_{k=0}^{m}$ be such that

$$
0=a_{0}=b_{0}<a_{1}<b_{1} \leq a_{2}<b_{2} \leq \cdots \leq a_{m}<b_{m}
$$

and

$$
\sum_{k=1}^{m} \frac{s_{k}-s_{k-1}}{b_{k}^{2}}<\frac{8}{\sigma^{2} \pi^{2}}
$$

Choose $r \in(0,1)$ such that

$$
0=a_{0}=r b_{0}<a_{1}<r b_{1} \leq a_{2}<r b_{2} \leq \cdots \leq a_{m}<r b_{m}
$$

and

$$
\frac{1}{r^{2}} \sum_{k=1}^{m} \frac{s_{k}-s_{k-1}}{b_{k}^{2}}<\frac{8}{\sigma^{2} \pi^{2}}
$$

Next pick $0<\delta<\min \left\{a_{1}, 1\right\}$ such that both

$$
\delta r\left(b_{k}-b_{k-1}\right)<(1+\delta) a_{k}<(1-\delta) r b_{k}, \quad 1 \leq k \leq m,
$$


and

$$
\frac{1}{r^{2}(1-\delta)^{2}} \sum_{k=1}^{m} \frac{s_{k}-s_{k-1}}{b_{k}^{2}}<\frac{8}{\sigma^{2} \pi^{2}}
$$

Also, pick $T \in(0,1)$ such that

$$
\frac{T}{r^{2}(1-\delta)^{2}} \sum_{k=1}^{m} \frac{s_{k}-s_{k-1}}{b_{k}^{2}}+\frac{1-T}{r^{2} \delta^{2}} \sum_{k=1}^{m} \frac{s_{k}-s_{k-1}}{\left(b_{k}-b_{k-1}\right)^{2}}<\frac{8}{\sigma^{2} \pi^{2}} .
$$

Throughout, let $\tau_{n}=n^{-n}, n=1,2,3, \ldots$, and observe that

$$
\lim _{n \rightarrow \infty} \frac{\tau_{n+1}}{\tau_{n}}=0
$$

Recall (4.3). As $V(\tau) \geq \sigma^{2}>0$ for all $\tau>0$, it follows from (4.31) that there exists $n_{0} \geq 1$ such that

$$
\sup _{n \geq n_{0}} \sqrt{\tau_{n}}\left|K\left(\sqrt{\tau_{n}}\right)\right| \sqrt{s_{m}} \leq \sup _{n \geq n_{0}} \frac{\sqrt{\tau_{n}}\left|\nu\left(\sqrt{\tau_{n}}\right)\right| \sqrt{s_{m}}}{\sigma} \leq 1 .
$$

In view of (5.15) there exists $n_{1} \geq n_{0}$ such that $\tau_{n+1}<s_{1} \tau_{n}$ for all $n \geq n_{1}$. For $n \geq n_{1}$ let $A_{n}$ be the event for which

$$
\sup _{\tau_{n+1}<u \leq \tau_{n} s_{k}}\left|\frac{X^{\left(\sqrt{\tau_{n}}\right)}(u)-X^{\left(\sqrt{\tau_{n}}\right)}\left(\tau_{n+1}\right)+v\left(\sqrt{\tau_{n}}\right)\left(u-\tau_{n+1}\right)}{\sqrt{\tau_{n} / \ell_{2}\left(\tau_{n}\right)}}\right| \in\left(a_{k}, r b_{k}\right],
$$

for all $1 \leq k \leq m$.

Let $C$ be the universal constant in Proposition 4.2. For $1 \leq k \leq m$ and $n \geq n_{1}$ set

$$
\begin{aligned}
\Delta s_{k} & =s_{k}-s_{k-1}, \\
\Delta s_{m}^{*} & =\min \left\{\Delta s_{k}: 1 \leq k \leq m\right\}, \\
D_{m} & =1+\frac{b_{m}}{\sigma \sqrt{\Delta s_{m}^{*}}},
\end{aligned}
$$

and

$$
a_{n, k}=\frac{a_{k}}{\sqrt{\ell_{2}\left(\tau_{n}\right) V\left(\sqrt{\tau_{n}}\right) \Delta s_{k}}}, \quad b_{n, k}=\frac{r b_{k}}{\sqrt{\ell_{2}\left(\tau_{n}\right) V\left(\sqrt{\tau_{n}}\right) \Delta s_{k}}} .
$$


Then we get from Proposition 4.2, (5.12), (5.13), and (5.16), that

$$
\begin{aligned}
P\left(A_{n}\right)= & P\left(\left\|Y^{\left(\sqrt{\tau_{n}}\right)}\right\|_{s_{k} \tau_{n}-\tau_{n+1}} \in \sqrt{\frac{\tau_{n}}{\ell_{2}\left(\tau_{n}\right) V\left(\sqrt{\tau_{n}}\right)}}\left(a_{k}, r b_{k}\right], \quad 1 \leq k \leq m\right) \\
\geq & \prod_{k=1}^{m} \Theta\left((1+\delta) a_{n, k},(1-\delta) b_{n, k}, \delta\left(b_{n, k}-b_{n, k-1}\right), T\right) \\
& -\frac{m C}{1-T}\left[\frac{\sqrt{s_{m}}}{\sigma} \sqrt{\tau_{n}}\left|\nu\left(\sqrt{\tau_{n}}\right)\right|+\frac{1}{\sigma^{3} \sqrt{\Delta s_{m}^{*}}} \frac{\rho\left(\sqrt{\tau_{n}}\right)}{\sqrt{\tau_{n}}} D_{m}\right],
\end{aligned}
$$

for all $n \geq n_{1}$. It follows from (4.31) that

$$
\sum_{n \geq n_{1}}\left[\frac{\sqrt{s_{m}}}{\sigma} \sqrt{\tau_{n}}\left|v\left(\sqrt{\tau_{n}}\right)\right|+\frac{1}{\sigma^{3} \sqrt{\Delta s_{m}^{*}}} \frac{\rho\left(\sqrt{\tau_{n}}\right)}{\sqrt{\tau_{n}}} D_{m}\right]<\infty .
$$

Recall that $V\left(\sqrt{\tau_{n}}\right) \downarrow \sigma^{2}>0$ as $n \rightarrow \infty$. By Lemma 4.4 and (5.14) we have

$$
\begin{aligned}
& \lim _{n \rightarrow \infty} \frac{1}{\ell_{2}\left(\tau_{n}\right)} \log \prod_{k=1}^{m} \Theta\left((1+\delta) a_{n, k},(1-\delta) b_{n, k}, \delta\left(b_{n, k}-b_{n, k-1}\right), T\right) \\
& =-\frac{\pi^{2} \sigma^{2}}{8}\left[\frac{T}{r^{2}(1-\delta)^{2}} \sum_{k=1}^{m} \frac{\Delta s_{k}}{b_{k}^{2}}+\frac{1-T}{r^{2} \delta^{2}} \sum_{k=1}^{m} \frac{\Delta s_{k}}{\left(b_{k}-b_{k-1}\right)^{2}}\right]>-1,
\end{aligned}
$$

and, thus,

$$
\sum_{n \geq n_{1}} \prod_{k=1}^{m} \Theta\left((1+\delta) a_{n, k},(1-\delta) b_{n, k}, \delta\left(b_{n, k}-b_{n, k-1}\right), T\right)=\infty .
$$

We, thus, get from (5.17) to (5.19) that

$$
\sum_{l \geq 0} P\left(A_{n+2 l}\right)+\sum_{l \geq 0} P\left(A_{n+2 l+1}\right)=\sum_{l \geq n}^{\infty} P\left(A_{l}\right)=\infty, \quad n \geq n_{1} .
$$

In view of (5.15), there exists $n_{2} \geq n_{1}$ such that $\tau_{n+2} s_{m}<\tau_{n+1}$ for all $n \geq n_{2}$. Consequently, both $\left(A_{n_{2}+2 l}\right)_{l \geq 0}$ and $\left(A_{n_{2}+2 l+1}\right)_{l \geq 0}$ are sequences of independent events. It, thus, follows from the Borel-Cantelli lemma and (5.20) that

$$
\begin{aligned}
& P\left(A_{n} \text { i.o. as } n \rightarrow \infty\right) \\
& \quad \geq P\left(A_{n_{2}+2 l} \text { i.o. as } l \rightarrow \infty\right) \vee P\left(A_{n_{2}+2 l+1} \text { i.o. as } l \rightarrow \infty\right)=1 .
\end{aligned}
$$

Next recall (4.4). As (4.31) is in place for $\tau_{n}=n^{-n}$ it follows from Lemma 4.1 that

$$
P\left(\left\|W^{\left(\sqrt{\tau_{n}}\right)}\right\|_{s_{m} \tau_{n}}>0, \quad \text { i.o. as } n \rightarrow \infty\right)=0,
$$


and, thus, by (5.21), with probability 1 ,

$$
\bigcap_{k=1}^{m}\left\{\frac{\sup _{\tau_{n+1} \leq u \leq \tau_{n} s_{k}}\left|X(u)-X\left(\tau_{n+1}\right)\right|}{\sqrt{\tau_{n} / \ell_{2}\left(\tau_{n}\right)}} \in\left(a_{k}, r b_{k}\right]\right\} \quad \text { i.o. as } n \rightarrow \infty \text {. }
$$

By the law of the iterated logarithm for Lévy processes at small times (cf. Sato ([30], Proposition 47.11), we have

$$
\limsup _{n \rightarrow \infty} \frac{\|X\|_{\tau_{n+1}}}{\sqrt{2 \tau_{n+1} \ell_{2}\left(\tau_{n+1}\right)}} \leq \limsup _{t \downarrow 0} \frac{|X(t)|}{\sqrt{2 t \ell_{2}(t)}}=\sigma, \quad \text { a.s. },
$$

and, thus, by (5.15),

$$
\lim _{n \rightarrow \infty} \frac{\|X\|_{\tau_{n+1}}}{\sqrt{\tau_{n} / \ell_{2}\left(\tau_{n}\right)}}=0, \quad \text { a.s. }
$$

Observe that

$$
\begin{aligned}
& \sup _{\tau_{n+1}<u \leq \tau_{n} s_{k}}\left|X(u)-X\left(\tau_{n+1}\right)\right|-\|X\|_{\tau_{n+1}} \\
& \leq\|X\|_{\tau_{n} s_{k}} \leq \sup _{\tau_{n+1}<u \leq \tau_{n} s_{k}}\left|X(u)-X\left(\tau_{n+1}\right)\right|+\|X\|_{\tau_{n+1}},
\end{aligned}
$$

for all $n \geq n_{1}$ and $1 \leq k \leq m$. Since $r \in(0,1)$ it, thus, follows from (5.22) to (5.24) that

$$
P\left(\mathcal{C}_{\left(n^{-n}\right)}(Z) \cap N^{m} \neq \emptyset\right)=1
$$

with $\mathcal{C}_{\left(n^{-n}\right)}$ as in (5.9) and

$$
N^{m}=\left\{g \in \mathcal{M}: a_{k}<g\left(s_{k}\right) \leq b_{k}, \quad 1 \leq k \leq m\right\} .
$$

Now the proof of the lemma is completed as follows: let $f \in(\sigma \pi / \sqrt{8}) \mathcal{K}$ and $\varepsilon>0$. By means of Lemma 4.6(iii) we can find a set $N \subseteq B_{\varepsilon}(f)=\left\{g \in \mathcal{M}: d_{\mathcal{M}}(f, g) \leq \varepsilon\right\}$ of the form (5.26), with $0=s_{0}<s_{1}<\cdots<s_{m}$ where $\left\{a_{k}\right\}_{k=0}^{m}$ and $\left\{b_{k}\right\}_{k=0}^{m}$ are sequences satisfying (5.10) and (5.11). By (5.25), we thus have $B_{\varepsilon}(f) \cap \mathcal{C}_{\left(n^{-n}\right)}(Z) \neq \emptyset$ a.s. for all $\varepsilon>0$. Further, since $\mathcal{C}_{\left(n^{-n}\right)}(Z)$ is closed, we have $\bigcap_{m=1}^{\infty}\left\{B_{1 / m}(f) \cap \mathcal{C}_{\left(n^{-n}\right)}(Z) \neq\right.$ $\emptyset\}=\left\{f \in \mathcal{C}_{\left(n^{-n}\right)}(Z)\right\}$ and, thus, for all $f \in(\sigma \pi / \sqrt{8}) \mathcal{K}, f \in \mathcal{C}_{\left(n^{-n}\right)}(Z)$, a.s. Hence $(\sigma \pi / \sqrt{8}) \mathcal{K} \subseteq \mathcal{C}_{\left(n^{-n}\right)}(Z)$ a.s. Since the inclusion $\mathcal{C}_{\left(n^{-n}\right)}(Z) \subseteq \mathcal{C}_{0}(Z)$ is immediate, the proof is complete.

Remark 5.1 In the situation of Theorem 2.1 we have $\mathcal{C}_{\left(n^{-n}\right)}(Z) \subseteq \mathcal{C}_{0}(Z)=\frac{\sigma \pi}{\sqrt{8}} \mathcal{K}$ a.s. In Lemma 5.2 we established that $\frac{\sigma \pi}{\sqrt{8}} \mathcal{K} \subseteq \mathcal{C}_{\left(n^{-n}\right)}(Z)$, a.s., and, thus, we even have the stronger result

$$
\mathcal{C}_{\left(n^{-n}\right)}(Z)=\mathcal{C}_{0}(Z)=\frac{\sigma \pi}{\sqrt{8}} \mathcal{K}, \quad \text { a.s. }
$$


At first it may seem surprising that $\mathcal{K}$ is revisited along such a sparse sequence. However, a similar observation was exploited by Jain and Pruitt [14] in their proof of (1.1).

It remains to show the relative compactness. Recall that a set $\mathcal{F} \subseteq \mathcal{M}$ is relatively compact in $\left(\mathcal{M}, d_{\mathcal{M}}\right)$ if and only if for all $\Gamma>0$ there exists $s>0$ such that $\inf _{f \in \mathcal{F}} f(s) \geq \Gamma$ [this follows from the construction of $d_{\mathcal{M}}$ and the well-known tightness criterion for compactness in $\mathcal{L}$ ].

Lemma 5.3 Let $T>0$. Assume $\sigma^{2}>0$ in (2.2). Then $Z=\left(Z_{t}\right)_{0<t \leq T}$ is relatively compact a.s.

Proof of Lemma 5.3 Let $\sigma^{2}>0$ in (2.2). In particular, $X$ is not the zero process and we have, for $P$-almost all $\omega \in \Omega$,

$$
\lim _{t \rightarrow \infty}\|X(\omega)\|_{t}=\infty
$$

By the same arguments as in [7] (cf. page 271), (3.2) follows from our Lemmas 5.1 and 5.2. Pick $\omega \in \Omega$, satisfying both (3.2) and (5.27). We claim that $\left(Z_{t}(\omega)\right)_{0<t \leq T}$ must then be relatively compact in $\mathcal{M}$.

To see this, let $\Gamma>0$ arbitrary. Let $s_{1}>0$ such that $\sqrt{s_{1}} \pi \sigma /(2 \sqrt{8}) \geq \Gamma$. Since $\ell_{2}(s t) / \ell_{2}(t) \rightarrow 1$ as $t \downarrow 0$ for all $s>0$, we get from (3.2) that

$$
\liminf _{t \downarrow 0} \frac{\|X(\omega)\|_{t s_{1}}}{\sqrt{t / \ell_{2}(t)}}=\sqrt{s_{1}} \liminf _{t \downarrow 0} \frac{\|X(\omega)\|_{t}}{\sqrt{t / \ell_{2}(t)}}=\frac{\sqrt{s_{1}} \pi \sigma}{\sqrt{8}},
$$

and, thus, there exists $\tau=\tau(\omega) \in(0, T)$ such that

$$
\inf _{0<t \leq \tau} \frac{\|X(\omega)\|_{t s_{1}}}{\sqrt{t / \ell_{2}(t)}} \geq \frac{\sqrt{s_{1}} \pi \sigma}{2 \sqrt{8}} \geq \Gamma .
$$

Since $\inf _{\tau \leq t \leq T}\|X(\omega)\|_{t s} / \sqrt{t / \ell_{2}(t)} \geq\|X(\omega)\|_{\tau s} / \sqrt{T}$, it follows from (5.27) that there exists $s_{2}=s_{2}(\omega) \geq s_{1}$ such that $\|X(\omega)\|_{\tau s_{2}} / \sqrt{T} \geq \Gamma$ and, thus,

$$
\inf _{0<t \leq T} Z_{t}\left(\omega, s_{2}\right)=\inf _{0<t \leq T} \frac{\|X(\omega)\|_{t s_{2}}}{\sqrt{t / \ell_{2}(t)}} \geq \inf _{0<t \leq \tau} \frac{\|X(\omega)\|_{t s_{1}}}{\sqrt{t / \ell_{2}(t)}} \wedge \frac{\|X(\omega)\|_{\tau s_{2}}}{\sqrt{T}} \geq \Gamma .
$$

$\left(Z_{t}(\omega)\right)_{0<t \leq T}$ is, thus, relatively compact in $\mathcal{M}$, provided $\omega$ satisfies (5.27) and (3.2). This completes the proof of the lemma.

Acknowledgments We are grateful to two referees for detailed, and helpful, comments.

\section{Appendix}

Proof of Lemma 4.6 We denote the set of continuity points of a function $g \in \mathcal{M}$ by $C(g)$. 
(i) It follows easily from Fatou's lemma that $\mathcal{K}$ is closed in $\mathcal{M}$. Observe that $\frac{S}{f^{2}(S)} \leq$ $\int_{0}^{S} \frac{\mathrm{d} z}{f^{2}(z)} \leq 1$ for all $S>0$ and $f \in \mathcal{K}$. Consequently, inf $f \in \mathcal{K} f(S) \geq \sqrt{S}$ for all $S>0$ and, thus, $\mathcal{K}$ must be relatively compact.

(ii) Let $f \in \mathcal{M} \backslash \mathcal{K}$. If there exists $x_{0}>0$ such that $f(x)=0$ for all $0 \leq x<x_{0}$ then the assertion is trivial. Otherwise, we have $\int_{0}^{\infty} \frac{\mathrm{d} z}{f^{2}(z)}>1$ with the integral converging in the indefinite Riemann sense. Consequently, there exist $d \geq 2$, and $\sigma_{1}, \ldots, \sigma_{d} \in C(f)$ such that $f\left(\sigma_{d}\right)<\infty$ and $0=\sigma_{0}<\sigma_{1}<\cdots<\sigma_{d}$ and $I(f):=\sum_{k=1}^{d} \frac{\sigma_{k}-\sigma_{k-1}}{f^{2}\left(\sigma_{k}\right)}>1$.

For $0<\varepsilon<\sigma_{1} / 2$ and $1 \leq k \leq d$ define $s_{2 k-1}(\varepsilon)=\sigma_{k}-2 \varepsilon, s_{2 k}(\varepsilon)=\sigma_{k}-\varepsilon$ and $s_{0}(\varepsilon)=0$ and

$$
\begin{aligned}
I_{\varepsilon}^{m}(f):= & \sum_{k=1}^{2 d-2} \frac{s_{k}(\varepsilon)-s_{k-1}(\varepsilon)}{\left(f\left(s_{k+1}(\varepsilon)\right)+\varepsilon\right)^{2}}+\frac{s_{2 d}(\varepsilon)-s_{2 d-2}(\varepsilon)}{\left(f\left(s_{2 d}(\varepsilon)\right)+\varepsilon\right)^{2}} \\
= & \frac{\sigma_{1}-2 \varepsilon}{\left(f\left(\sigma_{1}-\varepsilon\right)+\varepsilon\right)^{2}}+\sum_{k=2}^{d-1} \frac{\sigma_{k}-\sigma_{k-1}-\varepsilon}{\left(f\left(\sigma_{k}-\varepsilon\right)+\varepsilon\right)^{2}}+\sum_{k=1}^{d-1} \frac{\varepsilon}{\left(f\left(\sigma_{k+1}-2 \varepsilon\right)+\varepsilon\right)^{2}} \\
& +\frac{\sigma_{d}-\sigma_{d-1}}{\left(f\left(\sigma_{d}-\varepsilon\right)+\varepsilon\right)^{2}} .
\end{aligned}
$$

As $I_{\varepsilon}^{m}(f) \rightarrow I(f)>1$ as $\varepsilon \downarrow 0$ (recall $\sigma_{k} \in C(f)$ ), this completes the proof of (ii). (iii) For $h \in \mathcal{M}$ let $t_{\infty}(h)=\inf \{t>0: h(t)=\infty\}$ with the convention inf $\emptyset=\infty$. The proof of (iii) is completed by the following five steps:

First Step. (iii) holds exactly as stated for all $\varepsilon>0$ and $f \in \mathcal{K}$, strictly increasing. To see this, let $\varepsilon>0$ and $f \in \mathcal{K}$ be strictly increasing. Then there are $0=s_{0}<s_{1}$ $<\cdots<s_{m}$ and $\delta^{*}>0$ such that

$M_{s_{1}, \ldots, s_{m}, \delta^{*}}(f)=\left\{g \in \mathcal{M}: f\left(s_{k}\right)-\delta^{*}<g\left(s_{k}\right)<f\left(s_{k}\right)+\delta^{*}, \quad 1 \leq k \leq m\right\} \subseteq B_{\varepsilon}(f)$.

This is because the sets $M_{S_{1}, \ldots, s_{m}, \delta^{*}}(f)$ form a local basis of the neighbourhoods of $f$ in $\mathcal{M}$. As $f$ is strictly increasing we find $\delta \in\left(0, \delta^{*}\right)$ such that

$$
\begin{aligned}
0<a_{1} & :=f\left(s_{1}\right)-\delta<b_{1}:=f\left(s_{1}\right)+\delta \\
\leq a_{2} & :=f\left(s_{2}\right)-\delta<b_{2}:=f\left(s_{2}\right)+\delta \leq \cdots \leq \\
& \leq a_{m}:=f\left(s_{m}\right)-\delta<b_{m}:=f\left(s_{m}\right)+\delta .
\end{aligned}
$$

By construction, $\left\{g \in \mathcal{M}: a_{k}<g\left(s_{k}\right) \leq b_{k}, 1 \leq k \leq m\right\} \subseteq M_{s_{1}, \ldots, s_{m}, \delta^{*}}(f) \subseteq$ $B_{\varepsilon}(f)$ and

$$
\sum_{k=1}^{m} \frac{s_{k}-s_{k-1}}{b_{k}^{2}} \leq \sum_{k=1}^{m} \frac{s_{k}-s_{k-1}}{f^{2}\left(s_{k}\right)}<\int_{0}^{\infty} \frac{\mathrm{d} z}{f^{2}(z)} \leq 1
$$

This completes the proof of the first step. 
Second Step. For all $\varepsilon>0$ and $f \in \mathcal{K}$ with $t_{\infty}(f)=\infty$ there exists $g \in \mathcal{K} \cap B_{\varepsilon}(f)$, strictly increasing. To see this, let $f \in \mathcal{K}$ such that $t_{\infty}(f)=\infty$ and, for $\delta>0$, define $g_{\delta} \in \mathcal{M}$ by setting $g_{\delta}(x)=f(x)+\delta x$ for $x \geq 0$. For all $\delta>0, g_{\delta}$ is strictly increasing and, also, we have $g_{\delta} \in \mathcal{K}$. Since $g_{\delta} \rightarrow f$ in $\mathcal{M}$ as $\delta \downarrow 0$ this completes the proof of the second step.

Third Step. For all $\varepsilon>0$ and $f \in \mathcal{K}$ with $t_{\infty}(f) \in(0, \infty) \cap C(f)$ there exists $g \in \mathcal{K} \cap B_{\varepsilon}(f)$ with $t_{\infty}(g)=\infty$. Let $f \in \mathcal{K}$ with $t_{\infty}(f) \in(0, \infty) \cap C(f)$ and, for $0<\delta<t_{\infty}(f)$, define $g_{\delta} \in \mathcal{M}$ as follows: if $0 \leq x<t_{\infty}(f)-\delta$ then set $g_{\delta}(x)=f(x)$; otherwise, set

$$
g_{\delta}(x)=f\left(t_{\infty}(f)-\delta\right)+\frac{x}{\sqrt{\left(t_{\infty}(f)-\delta\right) \int_{t_{\infty}(f)-\delta}^{\infty} f^{-2}(z) \mathrm{d} z}}, \quad x \geq t_{\infty}(f)-\delta
$$

$g_{\delta}$ is well-defined and satisfies $t_{\infty}\left(g_{\delta}\right)=\infty$ for all $0<\delta<t_{\infty}(f)$. By construction, we have $g_{\delta} \in \mathcal{K}$ for all $0<\delta<t_{\infty}(f)$. It is clear that $g_{\delta}(x) \rightarrow f(x)$ as $\delta \downarrow 0$ for all $x<t_{\infty}(f)$. For all $x \geq t_{\infty}(f)$, we have $g_{\delta}(x) \geq f\left(t_{\infty}(f)-\delta\right) \uparrow \infty=f(x)$ as $\delta \downarrow 0$. This is because $t_{\infty}(f)$ is a continuity point of $f$. It, thus, follows that $g_{\delta} \rightarrow f$ in $\mathcal{M}$ as $\delta \downarrow 0$. This completes the proof of the third step.

Fourth Step. For all $\varepsilon>0$ and $f \in \mathcal{K}$ with $t_{\infty}(f) \in(0, \infty) \backslash C(f)$ there exists $g \in \mathcal{K} \cap$ $B_{\varepsilon}(f)$ with $t_{\infty}(g) \in(0, \infty) \cap C(g)$ : to see this, let $f \in \mathcal{K}$ with $t_{\infty}(f) \in(0, \infty) \backslash C(f)$ and, for $0<\delta<t_{\infty}(f)$, define $g_{\delta} \in \mathcal{M}$ as follows: if $0 \leq x<t_{\infty}(f)-\delta$ then set $g_{\delta}(x)=f(x)$; otherwise, if $x \geq t_{\infty}(f)$ then set $g_{\delta}(x)=\infty$. Finally, set

$$
g_{\delta}(x)=f(x)+\tan \left(\frac{\pi}{2 \delta}\left(x+\delta-t_{\infty}(f)\right)\right), \quad t_{\infty}(f)-\delta \leq x<t_{\infty}(f) .
$$

By construction we have $g_{\delta} \in \mathcal{K}$ and $t_{\infty}\left(g_{\delta}\right)=t_{\infty}(f) \in(0, \infty) \cap C\left(g_{\delta}\right)$ for all $0<\delta<t_{\infty}(f)$. Since $g_{\delta} \rightarrow f$ in $\mathcal{M}$ this settles the fourth step.

Fifth Step. For all $\varepsilon>0$ and $f \in \mathcal{K}$ with $t_{\infty}(f)=0$ there exists $g \in \mathcal{K} \cap B_{\varepsilon}(f)$ with $t_{\infty}(g) \in(0, \infty)$. Now $f=\infty 1_{(0, \infty)}$ is the only function $f \in \mathcal{M}$ with $t_{\infty}(f)=0$. In the same spirit as above, we may approximate $f$ by $g_{\delta} \in \mathcal{K}$ with $t_{\infty}\left(g_{\delta}\right) \in(0, \infty)$, for instance, take $g_{\delta}=\delta^{1 / 2} 1_{(0, \delta)}+\infty 1_{[\delta, \infty)}$ for $\delta>0$.

\section{References}

1. Anderson, T.W.: The integral of a symmetric unimodal function over a symmetric convex set and some probability inequalities. Proc. Am. Math. Soc. 6, 170-176 (1955)

2. Aurzada, F., Dereich, S.: Small deviations of general Lévy processes. Ann. Probab. 37, 20662092 (2009)

3. Bertoin, J.: Lévy Processes. Cambridge Tracts in Mathematics, vol. 121. Cambridge University Press, Cambridge (1996)

4. Bertoin, J., Doney, R.A., Maller, R.A.: Passage of Lévy processes across power law boundaries at small times. Ann. Probab. 36, 160-197 (2008)

5. Bingham, N.: Variants on the law the iterated logarithm. Bull. Lond. Math. Soc. 18, $433-467$ (1986)

6. Buchmann, B., Maller, R.A., Szimayer, A.: An almost sure functional limit theorem at zero for a class of Lévy processes normed by the square root function, and applications. Probab. Theory Relat. Fields 142, 219-247 (2008) 
7. Chen, X., Kuelbs, J., Li, W.: A functional LIL for symmetric stable processes. Ann. Probab. 28, 258276 (2000)

8. Chung, K.L.: On the maximum partial sums of sequences of independent random variables. Trans. Am. Math. Soc. 64, 205-233 (1948)

9. Csáki, E.: On the lower limits of maxima and minima of Wiener process and partial sums. Z. Wahrscheinlichkeitstheorie Und Verw. Gebiete 43, 205-221 (1978)

10. Csáki, E.: A relation between Chung's and Strassen's law of the iterated logarithm. Z. Wahrscheinlichkeitstheorie Und Verw. Gebiete 54, 287-301 (1980)

11. Einmahl, U.: On the other law of the iterated logarithm. Prob. Theory Relat. Fields 96, 97-106 (1993)

12. Einmahl, U., Mason, D.M.: A universal Chung-type law of the iterated logarithm. Ann. Probab. 22, 1803-1825 (1994)

13. Gorn, N., Lifshitz, M.: Chung's law and the Csáki function. Theor. Probab. 12, 399-420 (1999)

14. Jain, N.C., Pruitt, W.E.: The other law of the iterated logarithm. Ann. Probab. 3, 1046-1049 (1975)

15. Karatzas, I., Shreve, S.E.: Brownian Motion and Stochastic Calculus. Springer, New York (1991)

16. Kesten, H.: A universal form of the Chung-type law of the iterated logarithm. Ann. Probab. 25, 15881620 (1997)

17. Kuelbs, J., Li, W.V.: Metric entropy and the small deviation problem for Gaussian measures. J. Funct. Anal. 116, 113-157 (1993)

18. Kuelbs, J., Li, W.V.: A functional LIL and some weighted occupation measure results for fractional Brownian motion. J. Theor. Probab. 15, 1007-1030 (2002)

19. Li, W.V.: A Gaussian correlation inequality and its applications to small ball probabilities. Electron. Commun. Probab. 4, 111-118 (1999)

20. Li, W.V., Linde, W.: Approximation, metric entropy, and small ball estimates for Gaussian measures. Ann. Probab. 27, 1556-1578 (1999)

21. Li, W.V., Shao, Q.-M.: Gaussian processes: inequalities, small ball probabilities and applications. In: Rao, C.R., Shanbhag, D. (eds.) Stochastic Processes: Theory and Methods, Handbook of Statistics, vol. 19, pp. 533-597. Elsevier, New York (2001)

22. Linde, W., Shi, Z.: Evaluating the small deviation probabilities of subordinated Lévy processes. Stoch. Proc. Appl. 113, 273-287 (2004)

23. Lifshitz, M.A.: Bibliography of small deviation probabilities. Available from: http://www.proba. jussieu.fr/pageperso/smalldev/biblio.html

24. Maller, R.A.: Small-time versions of Strassen's law for Lévy processes. Proc. Lond. Math. Soc. 89, 531-558 (2009)

25. Mogul'skii, A.A.: Small deviations in a space of trajectories. Theory Probab. Appl. 19, 726-736 (1974)

26. Mueller, C.: Strassen's law for local time. Z. Wahrscheinlichkeitstheorie Und Verw. Gebiete 63, 29-41 (1983)

27. Mueller, C.: A connection between Strassen's and Donsker-Varadhan's laws of iterated logarithm. Z. Wahrscheinlichkeitstheorie Und Verw. Gebiete 87, 365-388 (1991)

28. N'Zi, M., Rémillard, B., Theorescu, R.: Between Strassen and Chung normalisations for Lévy's area process. Bernoulli 4, 115-125 (1998)

29. Rushton, J.: A functional LIL for $d$-dimensional stable processes; invariance for Lévy- and other weakly convergent processes. J. Theor. Probab. 20, 397-427 (2007)

30. Sato, K.: Lévy Processes and Infinitely Divisible Distributions. Cambridge University Press, Cambridge (1999)

31. Sakhanenko, A.I.: On the speed of convergence in a boundary problem. Theor. Probab. Appl. 19, 416421 (1974)

32. Savov, M.: Small time two-sided LIL behavior for Lévy processes at zero. Prob. Theory Relat. Fields 144, 74-98 (2009)

33. Shmileva, E.Yu.: Small ball probabilities for a centered Poisson process of high intensity. J. Math. Sci 128, 2656-2668 (2005)

34. Shmileva, E.Yu.: Small ball probabilities for jump Lévy processes from the Wiener domain of attraction. Stat. Probab. Lett. 76, 1873-1881 (2006)

35. Shmileva, E.Yu.: Shifted small deviations and Chung LIL for symmetric alpha-stable processes. Available from: http://arxiv.org/abs/0811.2583v2.pdf

36. Wichura, M.: A functional form of Chung's Law of the iterated logarithm for the maximum absolute partial sums, unpublished manuscript (1973) 